NISTIR 7950

\title{
Examination of the Impact of Fingerprint Spatial Area Loss on Matcher Performance in Various Mobile Identification Scenarios
}

Shahram Orandi

Kenneth Ko

Stephen S. Wood

John D. Grantham

Michael D. Garris

http://dx.doi.org/10.6028/NIST.IR.7950

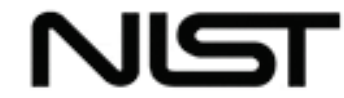

National Institute of Standards and Technology U.S. Department of Commerce 
NISTIR 7950

\title{
Examination of the Impact of Fingerprint Spatial Area Loss on Matcher Performance in Various Mobile Identification Scenarios
}

\author{
Shahram Orandi \\ Kenneth Ko \\ Stephen S. Wood \\ Michael D. Garris \\ Information Access Division \\ Information Technology Laboratory \\ John D. Grantham \\ Systems Plus, Inc. \\ Rockville, MD
}

http://dx.doi.org/10.6028/NIST.IR.7950

March 2014

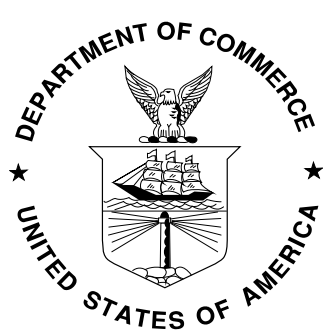

U.S. Department of Commerce

Penny Pritzker, Secretary

National Institute of Standards and Technology Patrick D. Gallagher, Under Secretary of Commerce for Standards and Technology and Director 


\section{Table of Contents}

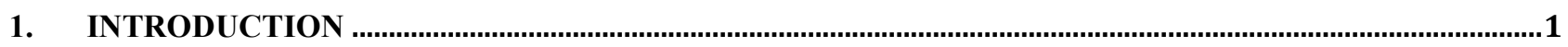

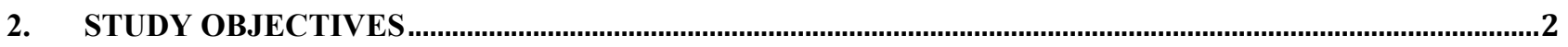

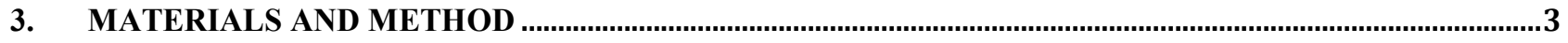

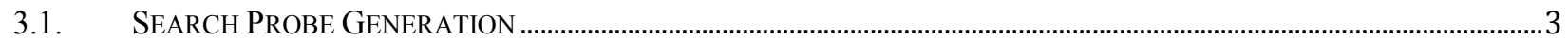

3.2. FINGER NUMBERING METHODOLOGY ………………………………………………………………………..

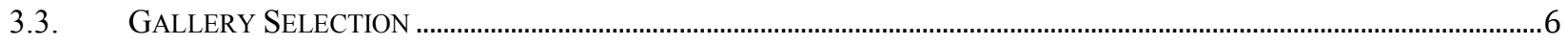

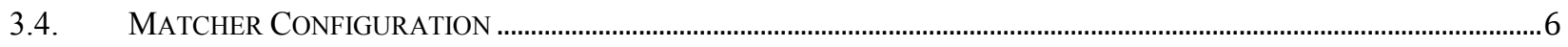

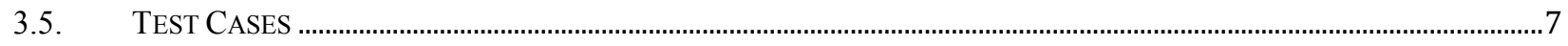

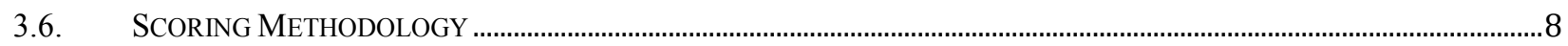

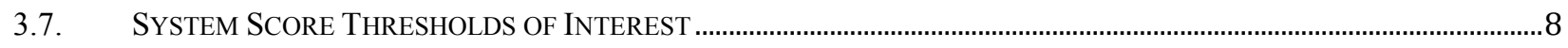

3.8. DISTANCE BASED COMPARISON AND RANKING ……................................................................................................

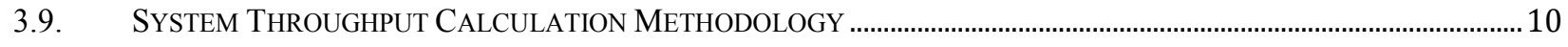

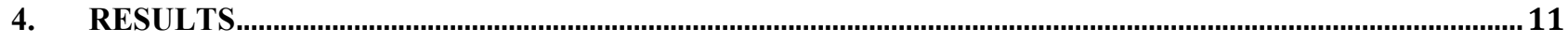

4.1. OBJECTIVE 1: BaSELINE IDFP SyStem PERFormanCE MEASUREMENT ……......................................................12

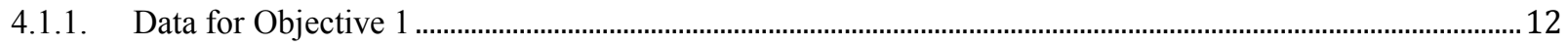

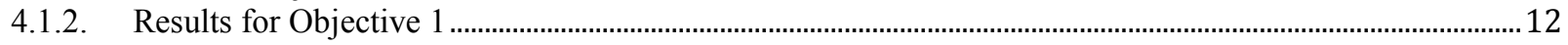

4.2. OBJECTIVE 2: TWO FINGER RISC SCENARIO COMPARISON ……..............................................................................13

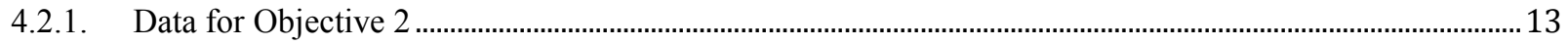

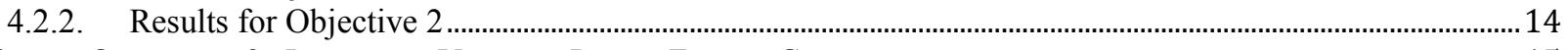

4.3. OBJeCtIVE 3: IMPACT OF VARIOUS PROBE FINGER COMBINATIONS ………………………................................15

4.3.1. $\quad$ Data for Objective 3 ................................................................................................................................... 15

4.3.2. Results for Objective 3 …………………........................................................................................... 17

4.4. OBJECTIVE 4: IMPACT OF GALLERY CONFIGURATION ON TWO FINGER FAPS.....................................................19

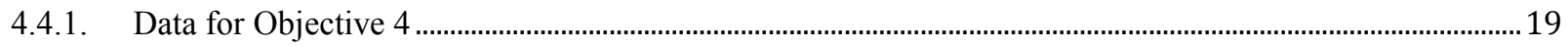

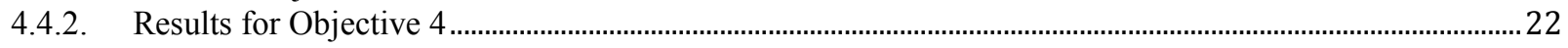

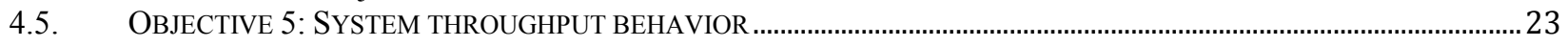

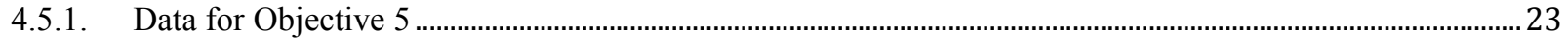

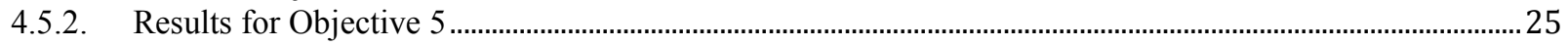

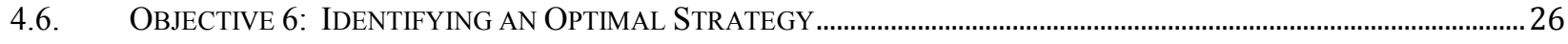

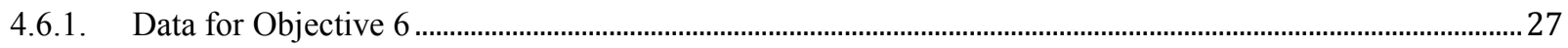

4.6.2. Results for Objective 6 .................................................................................................................................29

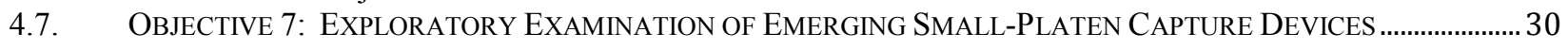

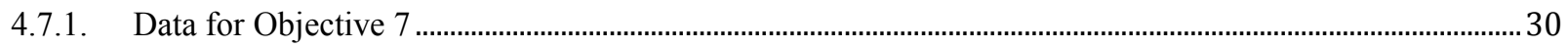

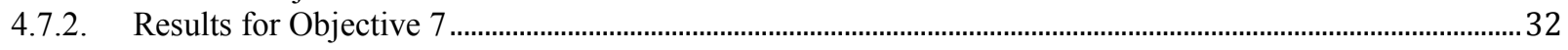

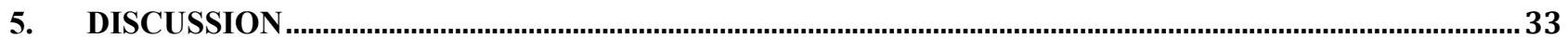

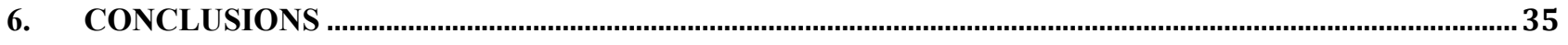

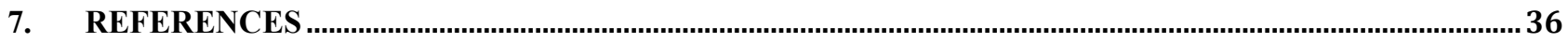




\section{List of Tables}

Table 1 - Acquisition Profiles Examined in this Study ...................................................................................................................

Table 2 - Simulated Mobile ID Data Generation Process ……………........................................................................................

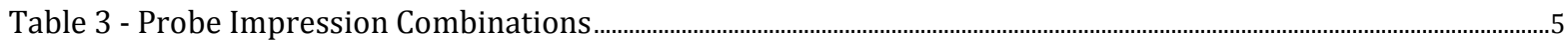

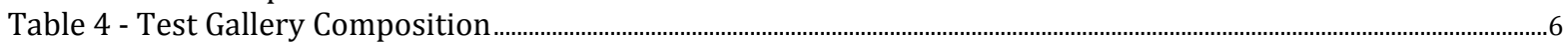

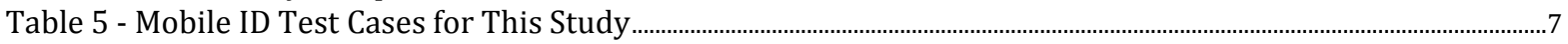

Table 6 - Scoring Threshold Values .............................................................................................................................................

Table 7 - Baseline IDFP System Performance...............................................................................................................................12

Table 8 - Matcher Performance by Mobile ID FAP, Two Finger Probe Scenario $(2,7)$........................................................13

Table 9 - System Performance by Various Probe Finger Combinations ...................................................................................15

Table 10 - System Performance by Various Probe Gallery Combinations, Two Finger Probe Scenario $(2,7) \ldots \ldots . .19$

Table 11 - Median System Matching Times (milliseconds) for Selected FAPs vs. FD-249 Gallery.............................23

Table 12 - Median Matching Times (milliseconds) for Image-Only Searches vs. Finger Combination........................24

Table 13 - Strictly Increasing FAP Median Match Times (FAP10 < FAP20 < FAP30) for Various Scenarios ...........25

Table 14 - Performance Data Ranked by Distance-to-Optimal for Error Trade-off ..............................................................2

Table 15 - Comparison of Experimental 1/4" FAP Versus Traditional Mobile ID FAPs......................................................31

Table 16 - Summary Matching Performance Data by Probe and Gallery Type.......................................................................38

Table 17 - Summary Matching Throughput by Probe and Gallery Type (median match time, milliseconds).........39

\section{List of Figures}

Figure 1 - Finger Numbering Methodology ...

Figure 2 - Matcher Performance by Mobile ID FAP, Two Finger Probe Scenario $(2,7)$.................................................13

Figure 3 - Error Tradeoff for Various Mobile ID FAPs in Two Finger (2, 7) Probes vs. FD-249 Gallery ...................14

Figure 4 - False Positive Identification Rates by Probe Finger Combination ..............................................................................16

Figure 5 - False Negative Identification Rates by Probe Finger Combination ..................................................................16

Figure 6 - Error Tradeoff for Various Mobile ID FAPs and Finger Combinations vs. FD-249 Gallery.........................16

Figure 7 - Error Tradeoff for Individual Mobile ID FAPs and Finger Combinations vs. FD-249 Gallery ....................17

Figure 8 - Matcher Performance by Gallery Configuration, Two Finger Probe Scenario $(2,7)$.....................................20

Figure 9 - Error Tradeoff for All Two Finger Mobile ID FAPs $(2,7)$ vs. Various Gallery Configurations....................21

Figure 10 - Error Tradeoff for Each Two Finger Mobile ID FAP $(2,7)$ vs. Various Gallery Configurations ...............22

Figure 11 - Median Matching Times (milliseconds) for Selected FAPs vs. FD-249 Gallery .............................................23

Figure 12 - Median Matching Times (milliseconds) for Image-Only Searches vs. Finger Combination......................24

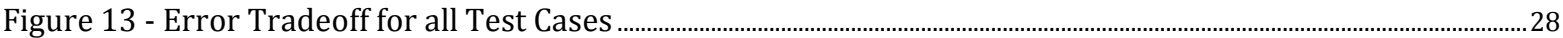

Figure 14 - Example of an Embedded Small Biometric Verification Sensor Such as the iPhone 5S............................30

Figure 15 - Error Tradeoff Comparison of Experimental 1/4" FAP Versus Traditional Mobile ID FAPs ........................31 


\section{Executive Summary}

NIST conducted an independent study to examine the impact of various mobile device Fingerprint Acquisition Profiles (FAPs) on the accuracy and throughput of the Next Generation Identification (NGI) Repository for Individuals of Special Concern (RISC) subsystem. RISC is intended for use primarily by personnel in the field operating portable or mobile devices that collect either fewer fingerprints than are traditionally captured in a fingerprint booking environment, and/or smaller fingerprint impressions than traditionally collected.

In conducting this study, NIST developed a database and a methodology which accommodates multiple enrollment gallery configurations as well as various spatial geometries for the probe images simulating the various fingerprint acquisition profiles according to the Mobile ID Best Practices Recommendation [MOBID] and the ANSI/NIST Standard [AN2k11] against various background (gallery) data scenarios.

For the typical field use-case of capturing two finger images and matching against a gallery of full FD-249 records, fingerprint acquisition profile 10 (FAP10) is at a disadvantage compared to other FAPs that define larger capture areas. In terms of false non-identification rate (FNIR), FAP10 submissions fail to identify their target more than twice as often as FAP20 (1.164 \% vs. $0.506 \%)$ at the system low confidence threshold (Yellow). For the high confidence threshold (Red), FNIR for FAP10 is almost twice that of FAP20 (3.270 \% vs. 1.844 \%). FAP30 on the other hand appears to be best/optimal and its performance is on a par with (if not slightly better than) the uncropped control case ( $d=0.0162$ for FAP30, $d=0.0164$ for control).

Comparing various probe finger combinations shows ten and four finger probe sets typically outperforming two finger probe sets in matching. While the FAP10 two finger operational case is not the worst performing among those tested, significant matching performance benefits can be realized by either increasing the count of fingers captured in FAP10, or utilizing FAP30 in two finger capture operations. Capturing more fingers using the smaller single-finger mobile capture devices may increase the risk of sequencing errors during the capture process so this mitigation strategy may not be optimal.

Examining system throughput performance shows that system match times appear closely linked to image spatial geometry where median search times for all image search FAPs consistently increases from FAP10 to FAP30 (with certain isolated exceptions). The two finger case of $(2,7)^{1}$ appears to provide the best throughput performance of all cases examined, while the two finger case of $(1,6)$ incurs an unusually large throughput penalty.

Given the data in this study, the operationally typical case of FAP10 two finger $(2,7)$ suffers from a significant performance penalty in terms of FNIR, showing a miss-rate almost twice as high as FAP20 and FAP30. It is possible to reduce FNIR significantly by adopting either FAP20 or FAP30. In cases where investment in legacy equipment must be preserved, a possible mitigation strategy for this performance gap is to utilize four finger capture (i.e., 2,3,7,8) with the smaller FAP10, while taking steps to avoid an increase in sequencing errors.

Finally, the two thumbs $(1,6)$ combination appears to be at a significant disadvantage ranking below most of the other cases examined in terms of FNIR. This disadvantage is marked and is not substantially mitigated by using various gallery strategies or FAP levels, with the two thumbs $(1,6)$ FAP10 test cases alone occupying 6 of the 8 lowest ranked performance cases out of the 60 cases examined in total.

${ }^{1}$ For more information on the finger numbering system used in this study please refer to section 3.2 . 


\begin{abstract}
Background: NIST conducted a study of the FBI Repository for Individuals of Special Concern (RISC) system using various gallery and Mobile ID [MOBID] acquisition profile combinations to examine performance characteristics of the various profiles in terms of matching effectiveness and throughput.

Results: The predominant RISC operational case of Mobile ID FAP10 (fingerprint acquisition profile 10) using the left and right index fingers is at a marked disadvantage in terms of matcher performance compared to the larger FAP2 0 and FAP30 cases using the same fingers. In terms of false non-identification rate (FNIR), FAP10 submissions fail to identify their target approximately twice as often as FAP20 or FAP30. FAP30 appears to be best/optimal and its performance is on a par with (if not slightly better) than the uncropped control case. False positive identification rates remain relatively flat across all the cases examined.

Conclusion: System false non-identification rates suffer a significant performance penalty in the typical operational case of FAP10 two index finger $(2,7)$ capture. FNIR performance can be markedly improved by either adopting FAP20 or FAP30 capture. To preserve legacy FAP10 equipment, it may be possible to utilize four finger capture (i.e., 2,3,7,8) with the smaller FAP10 to help mitigate some of the large performance penalty incurred with FAP10 in two finger operation, but this may increase the risk of sequencing errors from additional fingers having to be captured. Utilizing additional fingers or larger FAPs can also help mitigate performance penalties when a database of only-rolled or only-flat fingerprint impressions is used rather than one populated with complete FD-249 records.
\end{abstract}

\title{
Keywords
}

Mobile ID; Capture Spatial Area; NGI RISC; Biometrics; FAP10; SAP10; FAP20; SAP20; FAP30; SAP30 


\section{Introduction}

In 2013, the National Institute of Standards and Technology (NIST) - in partnership with the Federal Bureau of Investigation (FBI) - conducted an independent study to examine the impact of various mobile device Fingerprint Acquisition Profiles (FAPs) on the accuracy and throughput of the Next Generation Identification (NGI) Repository for Individuals of Special Concern (RISC) subsystem. The results of this effort provide insight into current system performance and a baseline for future NGI incremental improvements. The RISC subsystem is geared to providing an enhanced capability to identify persons of heightened investigative interest or who present special risks to the public or to law enforcement personnel. RISC is intended for use primarily by personnel in the field operating portable or mobile devices that collect either fewer fingerprints than are traditionally captured in a fingerprint booking environment, and/or smaller fingerprint impressions than traditionally collected.

In conducting this study, NIST developed a database and a methodology which accommodates multiple enrollment gallery configurations as well as various spatial geometries for the probe images simulating the various RISC capture scenarios according to the Mobile ID Best Practices Recommendation [MOBID] and the ANSI/NIST Standard [AN2k11].

The background data used in this study consisted of operational data provided by the FBI. Moreover, the search data was originally selected through biographical means without the use of an AFIS one-to-many search protocol, but later verified through automated biometric verification. This mitigates the possibility of bias in data selection caused by automated one-to-many biometric searching as the primary selection process. 


\section{Study Objectives}

NIST's objectives for this study examine the effectiveness of various RISC scenarios and the impact of those scenarios on matcher system performance ${ }^{2}$. The specific goals and objectives are as follows:

1. Establish a baseline of matcher performance in standard ten-print mode of operation, using identification-flat probe data as the base case.

2. Examine differences in RISC matcher performance given two finger Mobile ID acquisition profiles.

3. Examine differences in RISC matcher performance given various Mobile ID finger combinations.

4. Examine the impact of gallery impression type on the effectiveness of Mobile ID matching.

5. Measure the impact of various Mobile ID scenarios on system throughput performance.

6. Propose an optimization strategy for the system given the examined test cases.

7. Conduct a cursory evaluation of emerging small platen devices and the impact of such devices on matching performance.

2 Any hardware and software products identified in this report were used in order to perform the evaluations of experimental objectives described in this document. In no case does any such identification imply recommendation or endorsement by the National Institute of Standards and Technology, nor does it imply that the products and equipment identified are necessarily the best available for the purpose. 


\section{Materials and Method}

In order to create the dataset of fingerprint images to be used in this experiment, selections were made from the FBI-provided Platinum Data Repository (PDR). The PDR contains 4225062 mated biometric records including a mix of full rolled FD $-249^{3}$ capture sets and identification-flat 4 capture sets. Of the 4225062 records, a total of 390268 represent matched pairs of records meaning that each pair consists of two distinct biometric records taken from the same individual.

\subsection{Search Probe Generation}

A total of 100000 image sets were randomly selected from the PDR to serve as probe (search) biometric record sets. Of these probes, 50000 samples were selected that had exactly one corresponding matched/mated record available in the gallery and 50000 were selected that did not have any corresponding match/mate record available.

The probe sets were all selected to be identification-flat image sets which had already been segmented by an automated process to identify the location of the individual finger images in the larger multi-finger slap image. No effort was made to conduct further ground-truth evaluation and verification of the segmentation process.

The 100000 mated and non-mated probe image sets were then processed to produce image sets intended to simulate the various Subject Acquisition Profiles or SAPs as defined in [MOBID]. It should be noted that this terminology has now been superseded in [AN2k11] where these profiles are now referred to as Finger Acquisition Profiles or FAPs. The FAPs of interest that were examined in the scope of this study are described in Table 1 below.

Table 1 - Acquisition Profiles Examined in this Study

\begin{tabular}{lcccc}
\hline Dimensions & \multicolumn{3}{c}{ FAP(SAP) Profile Name } \\
\hline Image Width & \multicolumn{1}{c}{ FAP10 } & FAP20 & FAP30 & Control \\
Image Height & $0.65^{\prime \prime}(16.51 \mathrm{~mm})$ & $0.8^{\prime \prime}(20.32 \mathrm{~mm})$ & $1.0^{\prime \prime}(25.4 \mathrm{~mm})$ & Full image height $\left(1.5^{\prime \prime}\right.$ or $\left.38.10 \mathrm{~mm} \mathrm{max}\right)$ \\
Sample & & & \\
& & &
\end{tabular}

To create the simulated Mobile ID FAP images, a subsection of the finger images were cropped according to dimensions of interest for a given FAP (see Table 1). The cut sub-section was positioned at the geometric center of the finger as identified by using the segmentation coordinates of each digit as provided in the PDR data set (see Table 2).

3 The FD-249 is a sample collection card typically used in U.S. criminal booking scenarios. It allows for the capture of all 10 rolled fingerprint images, and the corresponding left-slap (left-slap-4), right-slap (right slap-4), left thumb (plain) and right thumb (plain) impressions.

4 The identification-flat (or id flat) image set consists of captured left-slap, right-slap, and a slap capture of both thumbs captured together or thumbs captured individually.

5 Resolution values and scanner platen dimensions are specified in inches and pixels per inch (ppi) respectively throughout this document. This is based on widely used specification guidelines for such devices and imagery, and is accepted as common nomenclature within the industry. SI units for these will be presented only once. 


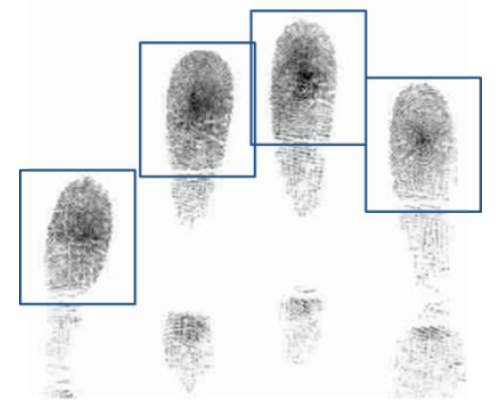

Stage 1 - Extract Segmentation Coordinates From Biometric Record Set

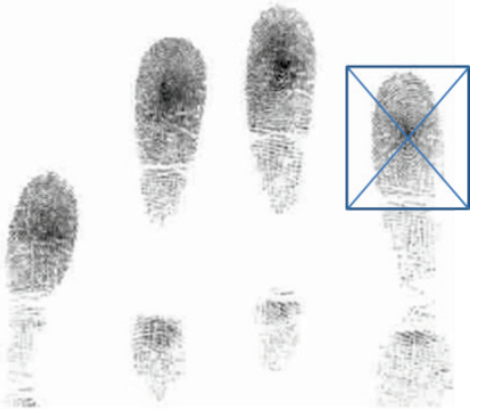

Stage 2 - Establish Geometric Center for Each Segmentation Box

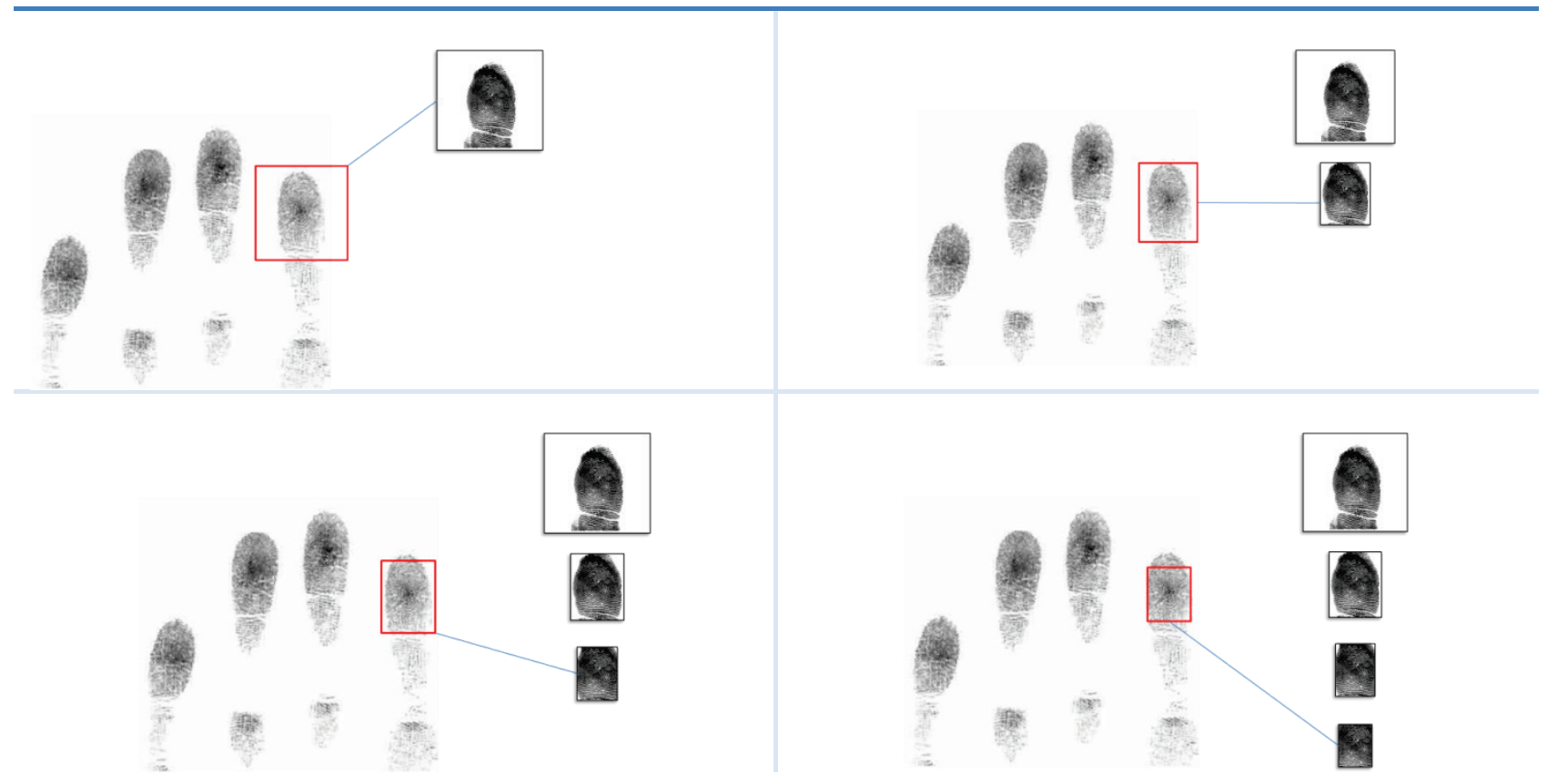

Stage 3 - Cut Image of Desired Size From Each Finger Image (Cut Location Centered by Segmentation Coordinates)

For FAP10, FAP20 and FAP30 when the segmentation box is smaller than the desired FAP dimensions, the cut subsection image is padded with white space (gray-scale index value of 255). The control image is an exception where no attempt is made to pad the image.

Given this data generation methodology, there is certain consistency in the way the simulated FAP10/20/30 images are cut and created for testing which can eliminate any bias from the variance in placement of the finger at each FAP capture. The variance in finger placement as they are presented naturally at capture time may have a measurable impact on matcher behavior as well that may be masked by the image cutting methodology in this study. Measuring the impact of finger placement however is outside of the scope of this study and may be explored in a separate study. 
In addition to various FAPs, probe sets were also configured for various finger impression combinations. These finger combinations are listed in Table 3.

Table 3 - Probe Impression Combinations

\begin{tabular}{|c|c|c|}
\hline Impression Case Number & Finger Numbers ${ }^{6}$ in Probe Set & Example \\
\hline 1 & 1 through 10 (full set) & \\
\hline 2 & $2,3,7,8$ & \\
\hline 3 & $1,2,6$ and 7 & \\
\hline 4 & 2 and 7 & \\
\hline 5 & 1 and 6 & \\
\hline
\end{tabular}

\section{2. $\quad$ Finger Numbering Methodology}

The finger numbering system used in this study is that of the Henry Classification System which is the accepted norm for the industry. In the Henry Classification system fingers on the right hand are numbered from 1 to 5 starting with the thumb, and moving outward to the small finger (see Figure 1). Fingers on the left hand are numbered from 6 to 10 starting with the thumb, and moving outward.

6 The finger numbering system used in this study is that of the Henry Classification System. For more information on this system please refer to section 3.2 . 


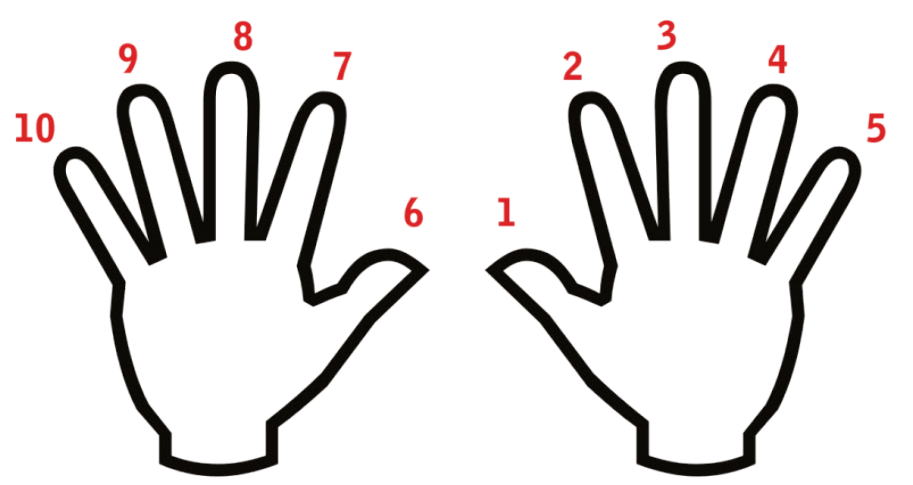

Figure 1 - Finger Numbering Methodology

\subsection{Gallery Selection}

A total of 1615228 FD-249 sets were selected from the PDR to serve as gallery image sets. Of these 1615228 image sets, 50000 sets had exactly one corresponding matched record set in the probe set and 1565228 were randomly selected so that they did not have any corresponding matching pair in the probe sets.

The 1615228 FD-249 records were then used to generate three discrete test gallery configurations sets (see Table 4). The first test gallery was populated to contain the original FD-249 full set with no changes or modifications. The second test gallery was populated by using only the rolled images from the FD-249 set (and omitting the flat/slap images). The third test gallery was populated by using only the flat/slap images (and omitting the rolled images) from the FD-249 base record.

During each test scenario execution, only one gallery configuration was active. The system was purged and repopulated with each test gallery to test new scenarios.

Table 4 - Test Gallery Composition

\begin{tabular}{ccc}
\hline Gallery Number & Gallery Composition & Gallery Size Total \\
\hline $\mathbf{1}$ & Original FD-249 set (Rolled and Flat Images) & 1615228 \\
$\mathbf{2}$ & Rolled Ten-Print Images Only & 1615228 \\
$\mathbf{3}$ & Slap (Flat) Ten-Print Images Only & 1615228 \\
\hline
\end{tabular}

The test gallery was generated with the intent to provide a robust test environment to yield measurement of matching errors with sufficient resolution for this study. The performance data obtained in this study therefore is dependent on the gallery selected for this study and may not be indicative of actual performance of the system using the contemporary FBI NGI gallery of over 70 million biometric records.

\subsection{Matcher Configuration}

The matcher used in this study is an FBI supplied modern state-of-industry commercial fingerprint matcher. The matcher supports both rolled fingerprints as well as flat fingerprint impression matching. The matcher also supports specific optimizations for data typical of Mobile ID in RISC field scenarios. 


\subsection{Test Cases}

Given the various combination of gallery and probe sets, a total of 60 test cases of interest were identified and executed for this study (see Table 5) and candidate lists with match scores were retrieved for each test case for scoring.

Table 5 - Mobile ID Test Cases for This Study

\begin{tabular}{|c|c|c|c|}
\hline Test Case Number & Gallery Configuration & Probe Type & Probe Impressions \\
\hline 1 & \multirow{17}{*}{ Full FD-249 } & \multirow{5}{*}{ FAP10 } & $1-10$ \\
\hline 2 & & & $2,3,7,8$ \\
\hline 3 & & & $1,2,6,7$ \\
\hline 4 & & & 2,7 \\
\hline 5 & & & 1,6 \\
\hline 6 & & \multirow{4}{*}{ FAP20 } & $1-10$ \\
\hline 7 & & & $2,3,7,8$ \\
\hline 8 & & & $1,2,6,7$ \\
\hline 10 & & & 1,6 \\
\hline 11 & & \multirow{5}{*}{ FAP30 } & $1-10$ \\
\hline 12 & & & $2,3,7,8$ \\
\hline 13 & & & $1,2,6,7$ \\
\hline 14 & & & 2,7 \\
\hline 15 & & & 1,6 \\
\hline 16 & & \multirow{3}{*}{ Control } & $1-10$ \\
\hline 17 & & & $2,3,7,8$ \\
\hline 20 & & & 1,6 \\
\hline 21 & \multirow{17}{*}{ Rolled Only } & \multirow{5}{*}{ FAP10 } & $1-10$ \\
\hline 22 & & & $2,3,7,8$ \\
\hline 23 & & & $1,2,6,7$ \\
\hline 24 & & & 2,7 \\
\hline 25 & & & 1,6 \\
\hline 26 & & \multirow{5}{*}{ FAP20 } & $1-10$ \\
\hline 27 & & & $2,3,7,8$ \\
\hline 28 & & & $1,2,6,7$ \\
\hline 29 & & & 2,7 \\
\hline 30 & & & 1,6 \\
\hline 31 & & \multirow{5}{*}{ FAP30 } & $1-10$ \\
\hline 32 & & & $2,3,7,8$ \\
\hline 33 & & & $1,2,6,7$ \\
\hline 34 & & & 2,7 \\
\hline 35 & & & 1,6 \\
\hline 39 & & \multirow{2}{*}{ Control } & 2,7 \\
\hline 40 & & & 1,6 \\
\hline 41 & \multirow{20}{*}{ Flat Only } & \multirow{5}{*}{ FAP10 } & $1-10$ \\
\hline 42 & & & $2,3,7,8$ \\
\hline 43 & & & $1,2,6,7$ \\
\hline 44 & & & 2,7 \\
\hline 45 & & & 1,6 \\
\hline 46 & & \multirow{5}{*}{ FAP20 } & $1-10$ \\
\hline 47 & & & $2,3,7,8$ \\
\hline 48 & & & $1,2,6,7$ \\
\hline 49 & & & 2,7 \\
\hline 50 & & & 1,6 \\
\hline 51 & & \multirow{5}{*}{ FAP30 } & $1-10$ \\
\hline 52 & & & $2,3,7,8$ \\
\hline 53 & & & $1,2,6,7$ \\
\hline 54 & & & 2,7 \\
\hline 55 & & & 1,6 \\
\hline 56 & & \multirow{5}{*}{ Control } & $1-10$ \\
\hline 57 & & & $2,3,7,8$ \\
\hline 58 & & & $1,2,6,7$ \\
\hline 59 & & & 2,7 \\
\hline 60 & & & 1,6 \\
\hline
\end{tabular}




\subsection{Scoring Methodology}

The test cases in this study were executed as an open-set 1:N (1 to $\mathrm{N}$, also known as one to many) matching scenario where a preset portion of subjects in the 1:N search actually have an enrolled mate in the gallery, and the probe set contains both mated and non-mated searches.

Mated searches are defined as probes for a given subject that is already enrolled in the gallery, and are expected to return a candidate list. Expected matches not returned, or returned with a score below the match score threshold are considered to be false negatives and are used in calculating the False Negative Identification Rate (FNIR). Given N enrolled subjects, with $\mathrm{L}$ candidates in the candidate list returned by the system using a threshold of $\mathrm{T}$ and rank of $\mathrm{R}$ (in this paper, $\mathrm{R}$ is always equal to 1 , and $\mathrm{L}$ is always equal to 20), FNIR is defined as:

Number of mate candidate searches where

$$
\operatorname{FNIR}(N, R, T, L)=\frac{\text { candidate's mate is below threshold } T \text { or below rank } R^{\text {Number of mate candidate lists, } S_{M}}}{\text { Number }}
$$

Non-mated searches are defined as probes for a given subject that has no corresponding mated record enrolled in the gallery, and searches using these non-mate probes should not return a candidate list with any score at or above the match score threshold T. Candidate lists returned with a score at or above the match threshold are considered to be false positives and are used in calculating the False Positive Identification Rate of this system (FPIR). Given N enrolled subjects, a candidate list of length L, and using a threshold of T, FPIR is defined as:

$$
\operatorname{FPIR}(N, T, L)=\frac{\begin{array}{c}
\text { Number of non }- \text { mate candidate lists with one or more } \\
\text { candidates at or above threshold } T
\end{array}}{\text { Number of non }- \text { mate candidate lists, } S_{N M}}
$$

\subsection{System Score Thresholds of Interest}

The system is designed and configured to support multiple decisional thresholds. These thresholds are designed to classify results of searches into categories that dictate a match success or failure, as well as candidates that would warrant further adjudication by additional processing steps such as human examiner review. 
For the purpose of this study, the RISC subsystem was measured according to the RISC performance thresholds listed below in Table 6.

Table 6 - Scoring Threshold Values

\begin{tabular}{|c|c|c|}
\hline Threshold Value & Threshold Name & Notes \\
\hline 2000 & RISC Low Confidence Hit & $\begin{array}{l}\text { A candidate match with a score at or above the low } \\
\text { confidence threshold, but below the high confidence } \\
\text { threshold, is considered a possible match, also referred to } \\
\text { as a "Yellow" hit ( ). } \\
\text { A candidate match with a score below the low confidence } \\
\text { threshold is considered a non-match, also referred to as a } \\
\text { "Green" non-hit (O). }\end{array}$ \\
\hline 5000 & RISC High Confidence Hit & $\begin{array}{l}\text { Typically, a candidate match with a score at or above a high } \\
\text { confidence threshold is considered a match with no further } \\
\text { adjudication or review necessary as part of the initial match } \\
\text { process. This is also referred to as a "Red" hit ( ). }\end{array}$ \\
\hline 12000 & IDFP7 Low Confidence Hit & $\begin{array}{l}\text { A score at or above the low confidence threshold on the } \\
\text { ten-print matching system indicates candidacy; however } \\
\text { some adjudication or review may be required. }\end{array}$ \\
\hline 25000 & IDFP High Confidence Hit & $\begin{array}{l}\text { A score at or above the high confidence threshold on the } \\
\text { ten-print matching system indicates a fully lights-out } \\
\text { candidacy decision with no adjudication, special processing } \\
\text { or reviews required. }\end{array}$ \\
\hline
\end{tabular}

\subsection{Distance Based Comparison and Ranking}

In addition to the individual performance measures of FNIR and FPIR, this study also utilizes a distance-based strategy for the comparison or ranking of performance by calculating a composite measure from FNIR and FPIR. This distance-based measure is defined as

$$
d=\sqrt{F N I R^{2}+F P I R^{2}}
$$

This formula is based on the calculation of distances in Euclidean space between the FPIR and FNIR value for a given test case to the Euclidian origin of $(0,0)$ which for error tradeoff corresponds to the optimal condition of $0 \%$ FPIR and $0 \%$ FNIR and represents the theoretical best-possible case. Therefore the lower the calculated $d$ is, the closer the test case in question is to the theoretical best-possible case, and the better the performance for that given case.

It should be noted that like data analysis methods involving Equal Error Rates (EER), the distance based method defined here treats both error types equally and does not apply any weights favoring either FPIR or FNIR.

7 The acronym IDFP or IdFP is commonly used to denote the FBI ten-print fingerprint identification system and associated processes. Although the IDFP system and the RISC system have basic design commonalities, they are quite distinct in terms of behavior, and have different characteristics. 


\subsection{System Throughput Calculation Methodology}

Due to the size of the candidate list diagnostic log files and the amount of computational resources required to capture, process and extract throughput data for all candidate lists (approximately 400 terabytes of data for all test cases in this study), it was not possible to generate and process diagnostic log files for all 100000 probes for each of the 60 test cases in the timeframe for this study. Instead, a subset of 6000 probes was randomly selected (3000 mates and 3000 non-mates) for each of the 60 cases examined, and timing data was calculated from this randomly selected subset.

The following caveats and conditions apply to the throughput portion of this study:

- Timing data was calculated as reported by the system for each candidate list returned in a diagnostic log file.

- System timing data was returned at a reported resolution of 1 milliseconds (ms) and inclusive of any/all processing overhead for each candidate search.

- No attempt was made to measure or remove I/O overhead resulting from normal system processing.

- The computational environment used for this study was isolated physically and logically from the general computing environment.

- No general processes were executed on the machines involved in this study while the throughput test was being conducted.

- System initialization and initial start-up times were not factored in measuring matcher performance. The test was commenced with the system fully initialized and at idle.

- The machines utilized for this study were time synchronized to a single machine within the environment. 


\section{Results}

The system gallery was initialized and probe sets were injected into the system for matching according to each of the 60 test case outlined in Table 5.

The resulting candidate lists for each test case were then scored according to the methodology described in section 3.6 and aggregate scores calculated where for each of the 60 test cases in Table 5, a set of FPIR and FNIR measurements were calculated for each of the scoring thresholds of interest described in Table 6.

A summary of all aggregate scores is provided in Table 16 of Appendix A. 


\subsection{Objective 1: Baseline IDFP System Performance Measurement}

The first objective of this study set out to establish how the system performs using identification-flat probes that have not been cropped in any way, and are matched against a standard full-set FD-249 gallery. This establishes baseline matcher performance inclusive of any bias that may be present in the data selected for this study.

The thresholds used for this examined case are specific to IDFP operation and differ from the RISC configuration; these IDFP thresholds are found in the third and fourth entries in Table 6.

All matcher-based RISC optimizations were disabled for this case to establish a baseline.

\subsubsection{Data for Objective 1}

Table 7 - Baseline IDFP System Performance

Test Parameter

Test Value

Case Description

Gallery Configuration

Gallery Size (Identities)

Control Case (IDFP, No RISC optimization)

Rolled and Flat (FD-249)

1615228

Probe Set Total (Identities, total count)

Probes (Mated count)

Probes (Non-Mated count)

100000

50000

50000

Probe Impression Type

ID Flat (Control, Not Cropped)

False Negative Identification Rate (FNIR)

False Positive Identification Rate (FPIR)

\begin{tabular}{|c|c|}
\hline Low Confidence Threshold (Yellow) & High Confidence Threshold (Red) \\
\hline $1.260 \%$ & $14.814 \%$ \\
$0.000 \%$ & $0.000 \%$ \\
\hline
\end{tabular}

Processing Time ${ }^{8}$ (Mated Probes)

Processing Time (Non-Mated Probes)

\begin{tabular}{|c|c|}
\hline Median Image Search Time (ms) & Median Template Search Time (ms) \\
\hline 3186 & 1739 \\
3096 & 1918 \\
\hline
\end{tabular}

\subsubsection{Results for Objective 1}

The system achieved an FNIR of $1.260 \%$ at the low confidence threshold (yellow) and $14.814 \%$ at the high confidence/lights-out threshold (red) set in this study. FPIR was at $0 \%$ for both thresholds (yellow and red) examined.

8 Throughput measurement testing was conducted using a subset of 6000 probes randomly selected from the 100000 probe set. See section 3.9 for more information. 


\subsection{Objective 2: Two Finger RISC Scenario Comparison}

The next objective in this study was to measure the effectiveness of the system matching performance when configured in RISC mode and operating on probe data meeting various Mobile ID finger acquisition profiles against a gallery of complete FD-249 image sets. The specific Mobile ID case examined for this objective is that of the two finger capture, typically from the left and right index fingers $(2,7)$ and is the predominant case for RISC in the field.

\subsubsection{Data for Objective 2}

Table 8 provides the data for this study objective and ranks the values from 1 (best) to 4 (worst) according to the distance-from-origin the methodology described in section 3.8 for each of the performance metrics (FPIR and FNIR), at both system confidence thresholds (yellow and red).

Table 8 - Matcher Performance by Mobile ID FAP, Two Finger Probe Scenario $(2,7)$

\begin{tabular}{|c|c|c|c|c|c|c|c|c|c|c|}
\hline 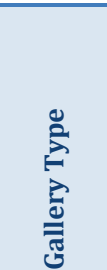 & 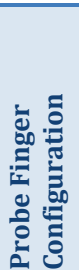 & 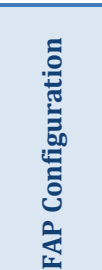 & 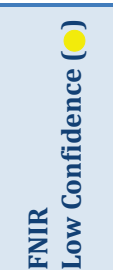 & 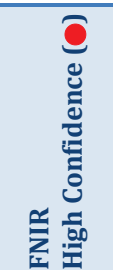 & 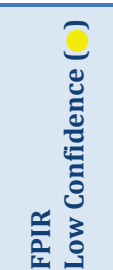 & 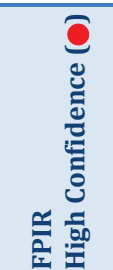 & 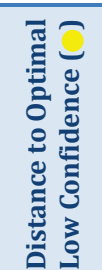 & 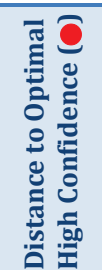 & 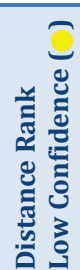 & 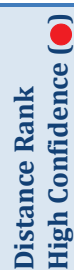 \\
\hline FD-249 & 2,7 & FAP30 & $0.400 \%$ & $1.616 \%$ & $0.356 \%$ & $0.000 \%$ & 0.0054 & 0.0162 & 1 & 1 \\
\hline FD-249 & 2,7 & control & $0.472 \%$ & $1.640 \%$ & $0.368 \%$ & $0.000 \%$ & 0.0060 & 0.0164 & 2 & 2 \\
\hline FD-249 & 2,7 & FAP20 & $0.506 \%$ & $1.844 \%$ & $0.422 \%$ & $0.000 \%$ & 0.0066 & 0.0184 & 3 & 3 \\
\hline FD-249 & 2,7 & FAP10 & $1.164 \%$ & $3.272 \%$ & $0.340 \%$ & $0.000 \%$ & 0.0121 & 0.0327 & 4 & 4 \\
\hline
\end{tabular}

Figure 2 shows summary data from Table 8 for the two print case of $(2,7)$ using a full FD-249 gallery with the matcher and with RISC optimizations enabled.

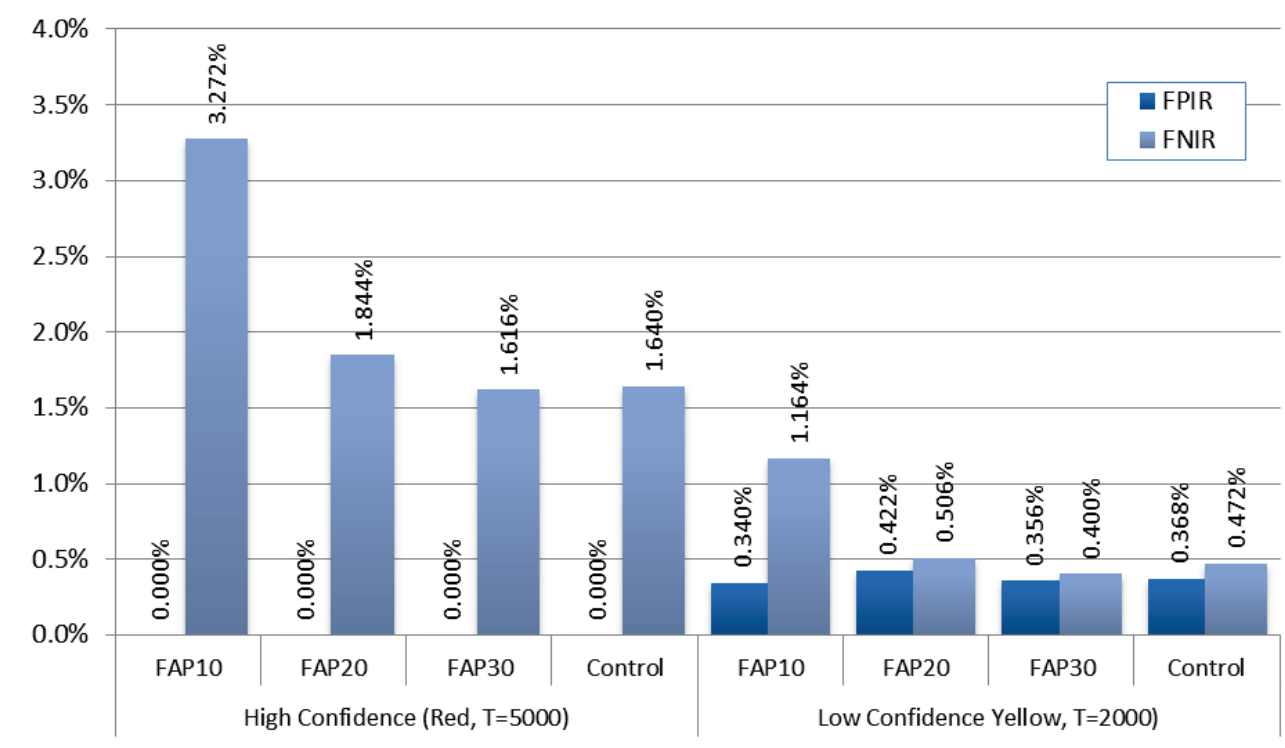

Figure 2 - Matcher Performance by Mobile ID FAP, Two Finger Probe Scenario $(2,7)$ 


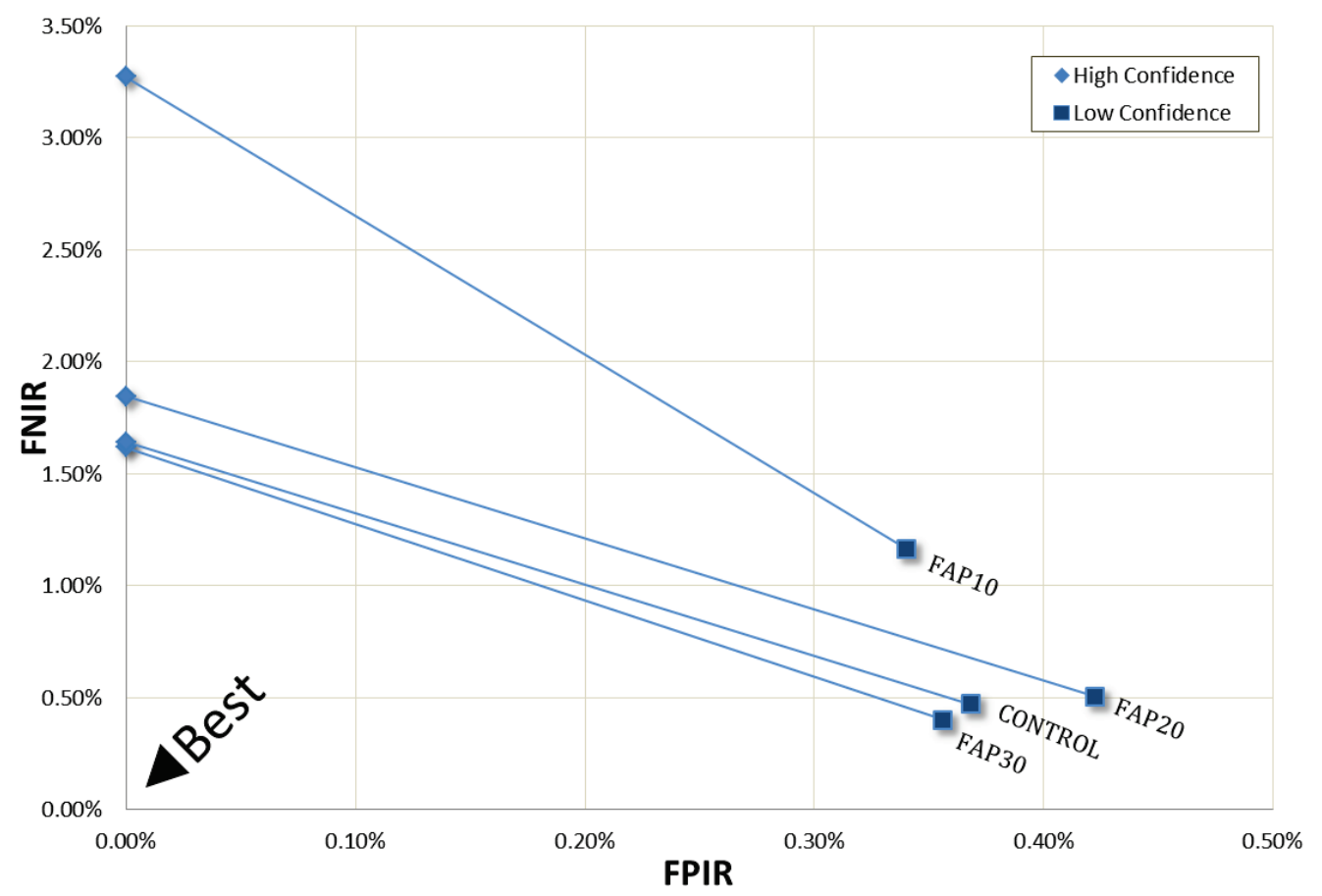

Figure 3 - Error Tradeoff for Various Mobile ID FAPs in Two Finger $(2,7)$ Probes vs. FD-249 Gallery

\subsubsection{Results for Objective 2}

For the two finger Mobile ID cases using the left and right index fingers (2, 7), FAP10 appears to be at a disadvantage when compared to other FAPs in terms of FNIR where FAP10 submissions fail to identify their mated target almost twice as often as FAP20 (1.164\% vs. $0.506 \%)$ at the Low Confidence system threshold (yellow hit). The same behavior holds in the high confidence system threshold (red hit) scenario where FNIR for FAP10 is almost twice as great as that of FAP20 (3.270\% vs. $1.844 \%)$. Error tradeoff analysis using the distance-from-origin methodology places the FAP10 case as last-ranked and worst for both low and high confidence FNIR, while FAP30 is best.

FPIR rates for all cases is flat at $0 \%$ at the high confidence threshold (red hit), and none of the Mobile ID FAPs show any disadvantage over the others in the high confidence scenario. For the low confidence system threshold scenario (yellow hit), the difference between matcher performance is very small with FAP10 showing a very slight advantage over FAP30 (0.340 \% vs. $0.356 \%$ ).

Using the distance-to-origin methodology to examine the error tradeoff between FPIR and FNIR shows that FAP30 excels over all other Mobile ID two finger $(2,7)$ FAPs.

What was surprising here was that FAP30 outperformed the control case, though by a very small margin. While this difference in performance is small (i.e., an FNIR of $1.616 \%$ vs $1.640 \%$ for the two finger $(2,7)$ case at the high confidence threshold) this difference is statistically significant $(\mathrm{p}<0.0001$ level, using Wilcoxon signed-rank test) and this pattern was observed in several other case clusters where FAP30 outperformed the control case. 


\subsection{Objective 3: Impact of Various Probe Finger Combinations}

The next objective in this study was to measure system matching performance when configured in RISC mode and using various combinations of fingers for Mobile ID searches against a gallery of complete FD-249 image sets. The finger combinations examined for this objective are described in Table 3.

\subsubsection{Data for Objective 3}

Table 9 summarizes the data from this objective utilizing all finger combinations described in Table 3 using a full FD-249 gallery with the matcher in RISC optimization mode, and ranks the values from 1 (best) to 20 (worst) using distance-to-optimal calculations (section 3.8) for each of the performance metrics (FPIR and FNIR) at both system confidence thresholds (High/Red and Low/Yellow). Figure 4 and Figure 5 show summary data from Table 9, for FPIR and FNIR respectively.

Table 9 - System Performance by Various Probe Finger Combinations

\begin{tabular}{|c|c|c|c|c|c|c|c|c|c|c|}
\hline 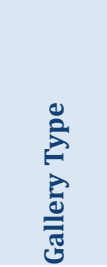 & 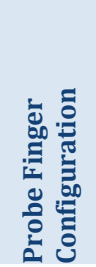 & 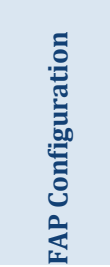 & 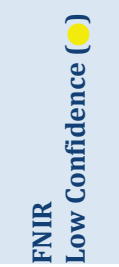 & 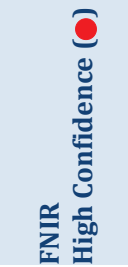 & 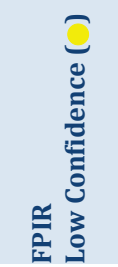 & 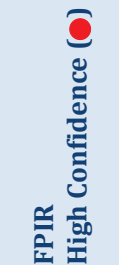 & 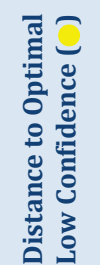 & 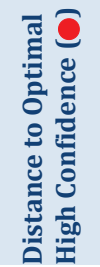 & 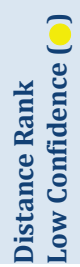 & 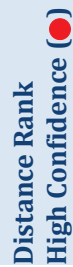 \\
\hline FD-249 & $1-10$ & control & $0.134 \%$ & $0.218 \%$ & $0.052 \%$ & $0.000 \%$ & 0.0014 & 0.0022 & 2 & 2 \\
\hline FD-249 & $1-10$ & FAP20 & $0.190 \%$ & $0.306 \%$ & $0.060 \%$ & $0.000 \%$ & 0.0020 & 0.0031 & 3 & 3 \\
\hline FD-249 & $2,3,7,8$ & FAP30 & $0.160 \%$ & $0.338 \%$ & $0.548 \%$ & $0.000 \%$ & 0.0057 & 0.0034 & 8 & 4 \\
\hline FD-249 & $2,3,7,8$ & control & $0.192 \%$ & $0.374 \%$ & $0.546 \%$ & $0.000 \%$ & 0.0058 & 0.0037 & 9 & 5 \\
\hline FD-249 & $1,2,6,7$ & FAP30 & $0.216 \%$ & $0.390 \%$ & $0.122 \%$ & $0.000 \%$ & 0.0025 & 0.0039 & 4 & 6 \\
\hline FD-249 & $1,2,6,7$ & control & $0.226 \%$ & $0.410 \%$ & $0.158 \%$ & $0.000 \%$ & 0.0028 & 0.0041 & 5 & 7 \\
\hline FD-249 & $2,3,7,8$ & FAP20 & $0.236 \%$ & $0.442 \%$ & $0.650 \%$ & $0.000 \%$ & 0.0069 & 0.0044 & 12 & 8 \\
\hline FD-249 & $1,2,6,7$ & FAP20 & $0.464 \%$ & $0.720 \%$ & $0.128 \%$ & $0.000 \%$ & 0.0048 & 0.0072 & 6 & 9 \\
\hline FD-249 & 2,7 & control & $0.472 \%$ & $1.640 \%$ & $0.368 \%$ & $0.000 \%$ & 0.0060 & 0.0164 & 10 & 11 \\
\hline FD-249 & $2,3,7,8$ & FAP10 & $1.372 \%$ & $1.714 \%$ & $0.622 \%$ & $0.000 \%$ & 0.0151 & 0.0171 & 14 & 12 \\
\hline FD-249 & 2,7 & FAP20 & $0.506 \%$ & $1.844 \%$ & $0.422 \%$ & $0.000 \%$ & 0.0066 & 0.0184 & 11 & 13 \\
\hline FD-249 & 2,7 & FAP10 & $1.164 \%$ & $3.272 \%$ & $0.340 \%$ & $0.000 \%$ & 0.0121 & 0.0327 & 13 & 14 \\
\hline FD-249 & 1,6 & control & $1.890 \%$ & $3.560 \%$ & $0.046 \%$ & $0.000 \%$ & 0.0189 & 0.0356 & 15 & 15 \\
\hline FD-249 & 1,6 & FAP30 & $1.964 \%$ & $3.598 \%$ & $0.090 \%$ & $0.000 \%$ & 0.0197 & 0.0360 & 16 & 16 \\
\hline FD-249 & $1-10$ & FAP10 & $3.740 \%$ & $3.940 \%$ & $0.034 \%$ & $0.000 \%$ & 0.0374 & 0.0394 & 19 & 17 \\
\hline FD-249 & $1,2,6,7$ & FAP10 & $3.658 \%$ & $4.040 \%$ & $0.146 \%$ & $0.000 \%$ & 0.0366 & 0.0404 & 18 & 18 \\
\hline FD-249 & 1,6 & FAP20 & $2.620 \%$ & $5.130 \%$ & $0.106 \%$ & $0.000 \%$ & 0.0262 & 0.0513 & 17 & 19 \\
\hline FD-249 & 1,6 & FAP10 & $5.938 \%$ & $10.402 \%$ & $0.208 \%$ & $0.000 \%$ & 0.0594 & 0.1040 & 20 & 20 \\
\hline
\end{tabular}




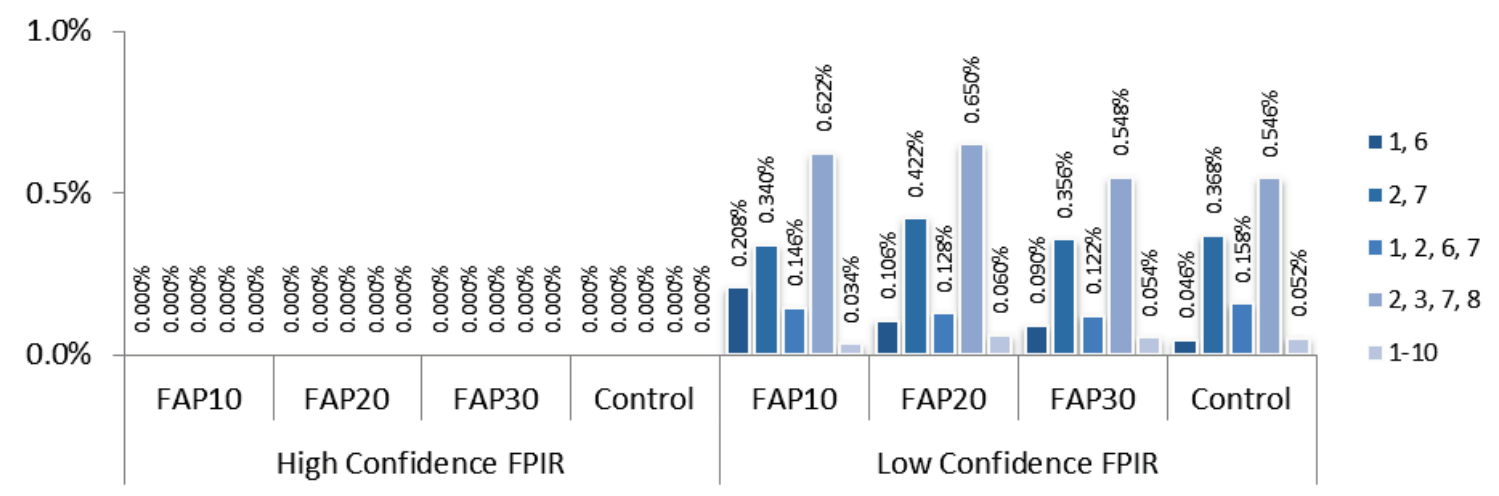

Figure 4 - False Positive Identification Rates by Probe Finger Combination

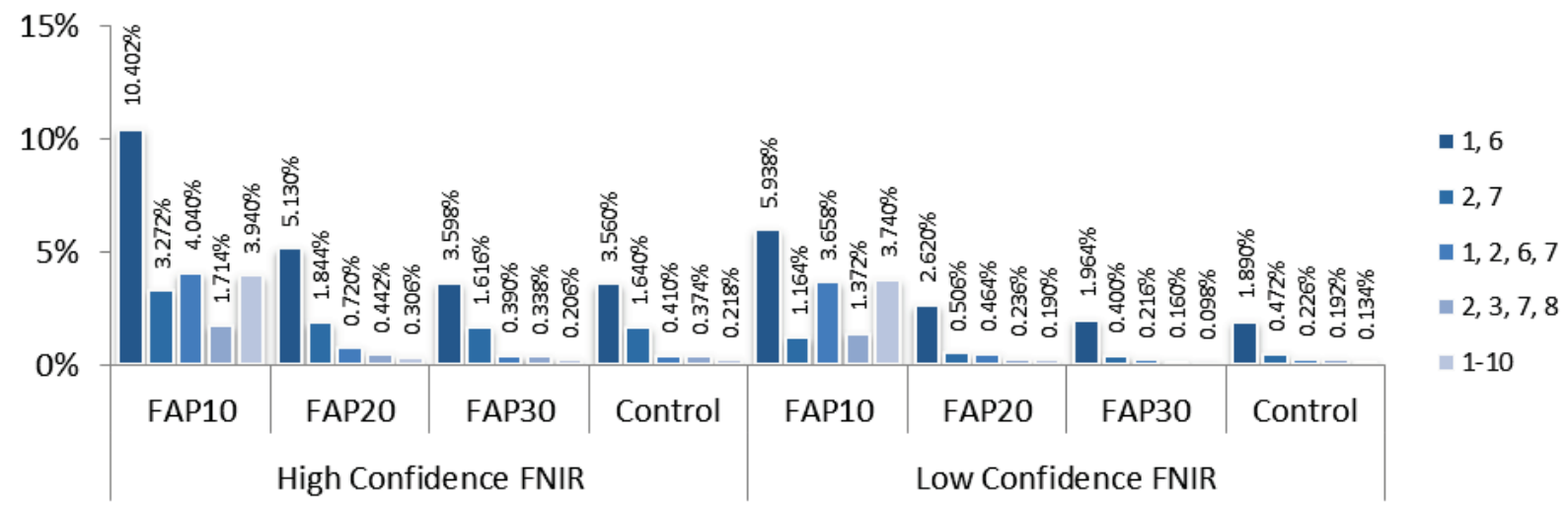

Figure 5 - False Negative Identification Rates by Probe Finger Combination

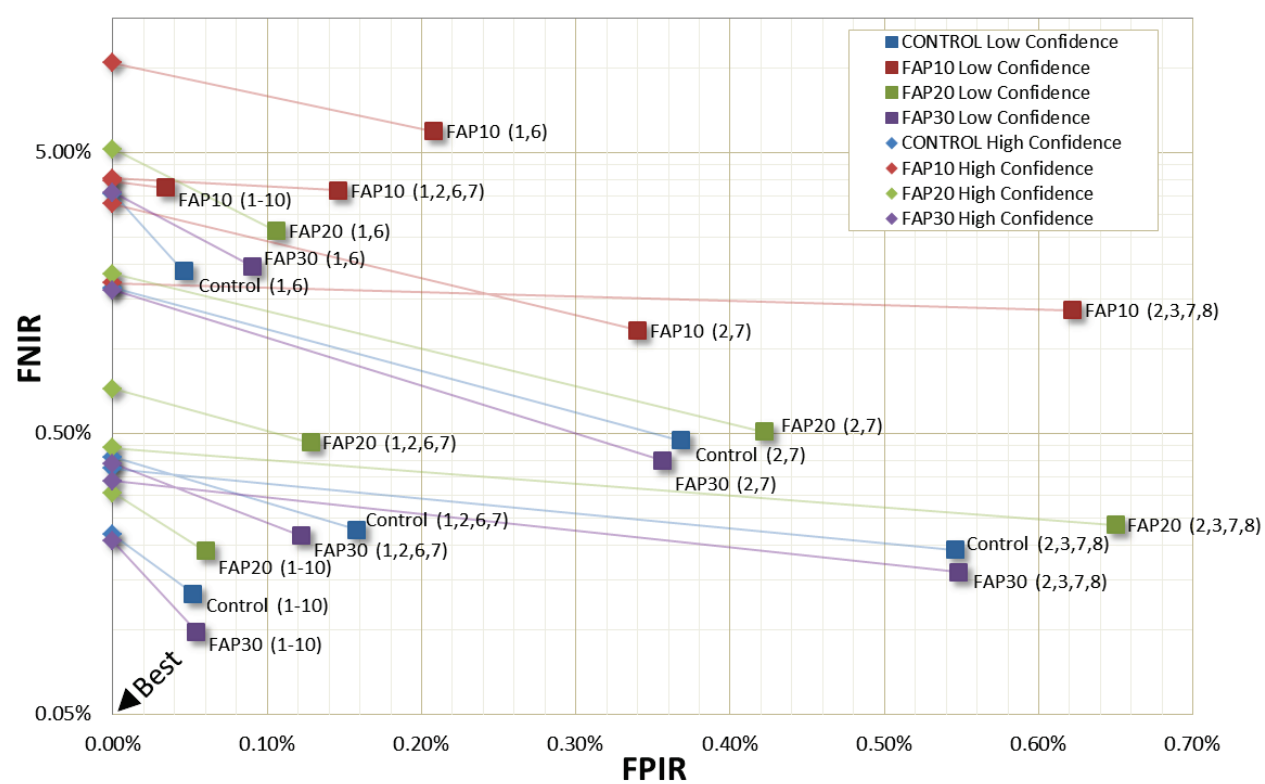

Figure 6 - Error Tradeoff for Various Mobile ID FAPs and Finger Combinations vs. FD-249 Gallery 


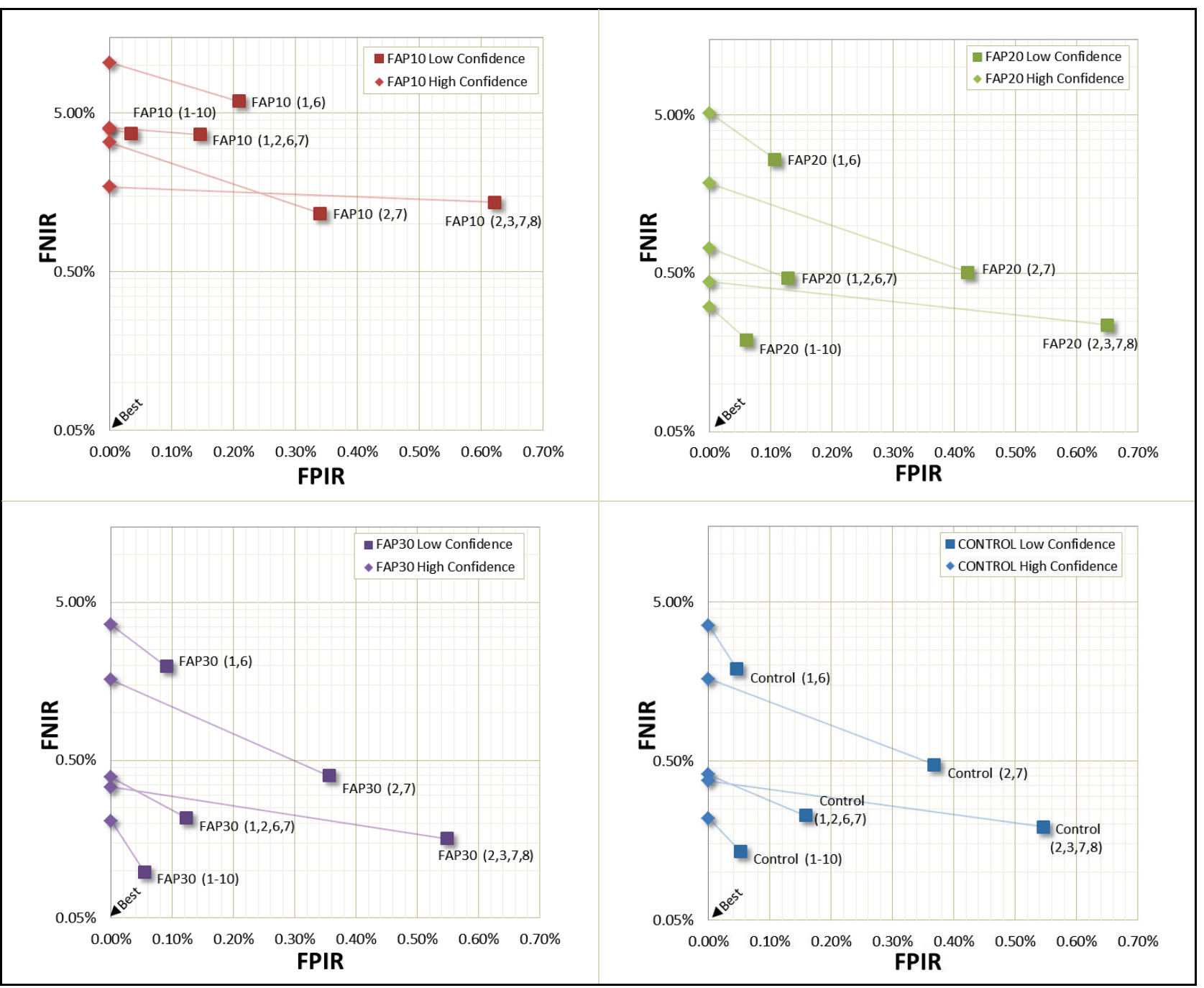

Figure 7 - Error Tradeoff for Individual Mobile ID FAPs and Finger Combinations vs. FD-249 Gallery

\subsubsection{Results for Objective 3}

The data from this study objective shows that FAP30 (1-10) finger case excels, followed by the Control (1-10) finger case, and the FAP20 (1-10) finger case when compared to a full FD-249 gallery, for both the high and low confidence thresholds.

On the other end of the scale, FAP10 $(1,6)$ two finger case ranks last in performance for both the high and low confidence thresholds.

At the high confidence system threshold FPIR appears flat at $0 \%$ for all cases and is not impacted by the various finger combination treatments. At the low confidence system threshold, FPIR does show some fluctuation but the fluctuation is generally small with all values below $1 \%$. 
FNIR performance appears widely impacted by the number of fingers and favors cases where the search probe had more finger impressions. FNIR at the high confidence system threshold ranges from $0.206 \%$ for the FAP30 (1-10) finger combination treatment, to $10.402 \%$ for the FAP10 $(1,6)$ finger combination treatment. To further emphasize the positive impact on the system's FNIR when additional fingers are used, it can be seen in Table 9 that 9 out of the 10 top-ranked cases were those that had four or ten finger probes.

FNIR performance at the low confidence threshold nearly mimics the high confidence performance, with the FNIR rates better than halved (i.e., $0.098 \%$ vs. $0.206 \%$ ) for the (1-10) FAP30 case which emerged as the best. 


\subsection{Objective 4: Impact of Gallery Configuration on Two Finger FAPs}

The next objective in this study was to measure system matching performance when configured in RISC mode and using various gallery combinations listed in Table 4 for the two finger $(2,7)$ combination case.

\subsubsection{Data for Objective 4}

Table 10 below provides data from tests utilizing the (2,7) finger combination for all Mobile ID FAPs described in Table 1 versus each gallery described in Table 4 with the matcher in RISC optimization mode. The data in Table 10 has been sorted according to rank values ranging from 1 (best) to 12 (worst) for each of the performance metrics (FPIR and FNIR) and both system confidence thresholds (High and Low). A summary graph is provided as Figure 8 as well as error trade-off charts Figure 9 (all FAPs in a single graph), and Figure 10 where error tradeoffs are provided separately for each FAP.

Table 10 - System Performance by Various Probe Gallery Combinations, Two Finger Probe Scenario (2, 7)

\begin{tabular}{|c|c|c|c|c|c|c|c|c|c|}
\hline 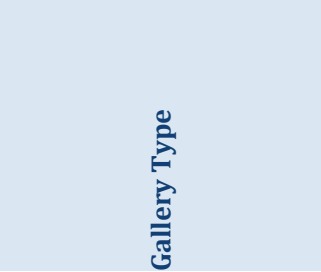 & 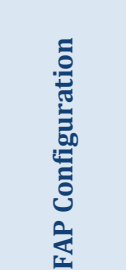 & 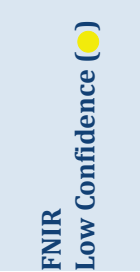 & 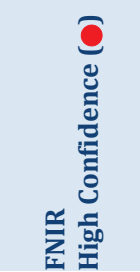 & 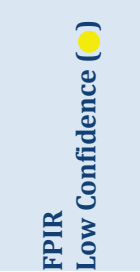 & 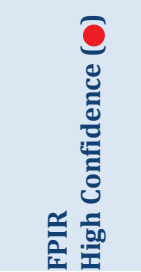 & 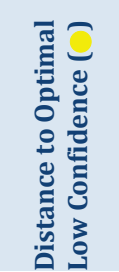 & 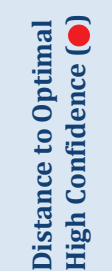 & 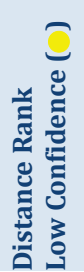 & 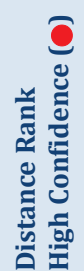 \\
\hline FD-249 Gallery & FAP30 & $0.400 \%$ & $1.616 \%$ & $0.356 \%$ & $0.000 \%$ & 0.0054 & 0.0162 & 1 & 1 \\
\hline FD-249 Gallery & Control & $0.472 \%$ & $1.640 \%$ & $0.368 \%$ & $0.000 \%$ & 0.0060 & 0.0164 & & \\
\hline FD-249 Gallery & FAP20 & $0.506 \%$ & $1.844 \%$ & $0.422 \%$ & $0.000 \%$ & 0.0066 & 0.0184 & 3 & 3 \\
\hline FD-249 Gallery & FAP10 & $1.164 \%$ & $3.272 \%$ & $0.340 \%$ & $0.000 \%$ & 0.0121 & 0.0327 & 4 & 4 \\
\hline Rolled-Only Gallery & FAP30 & $1.318 \%$ & $3.862 \%$ & $0.222 \%$ & $0.000 \%$ & 0.0134 & 0.0386 & 5 & 5 \\
\hline Flat-Only Gallery & FAP30 & $1.570 \%$ & $4.032 \%$ & $0.396 \%$ & $0.000 \%$ & 0.0162 & 0.0 & 7 & 6 \\
\hline Rolled-Only Gallery & Control & $1.588 \%$ & $4.078 \%$ & $0.284 \%$ & $0.000 \%$ & 51 & 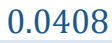 & 6 & 7 \\
\hline Flat-Only Gallery & Control & $1.726 \%$ & $4.138 \%$ & $0.406 \%$ & $0.000 \%$ & 0.0177 & 0.0 & 8 & 8 \\
\hline Flat-Only Gallery & FAP20 & $1.766 \%$ & $4.474 \%$ & $0.462 \%$ & $0.000 \%$ & 0.0183 & 0.0 & 10 & 9 \\
\hline Rolled-Only Gallery & FAP20 & $1.806 \%$ & $4.494 \%$ & $0.236 \%$ & $0.000 \%$ & 0.0182 & 0.0449 & 9 & 10 \\
\hline Flat-Only Gallery & FAP10 & $2.806 \%$ & $6.694 \%$ & $0.374 \%$ & $0.000 \%$ & 0.0283 & 0.0669 & 11 & 11 \\
\hline Rolled-Only Gallery & FAP10 & $4.024 \%$ & $8.048 \%$ & $0.294 \%$ & $0.000 \%$ & 0.0403 & 0.0805 & 12 & 12 \\
\hline
\end{tabular}




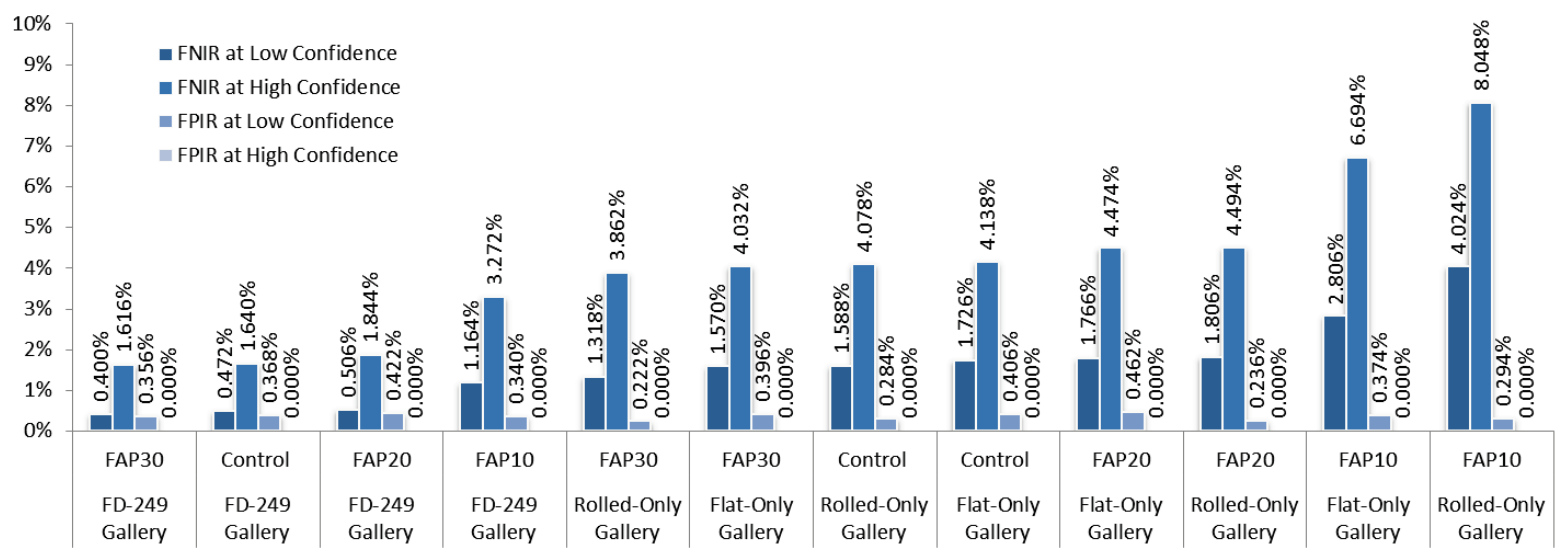

Figure 8 - Matcher Performance by Gallery Configuration, Two Finger Probe Scenario $(2,7)$ 


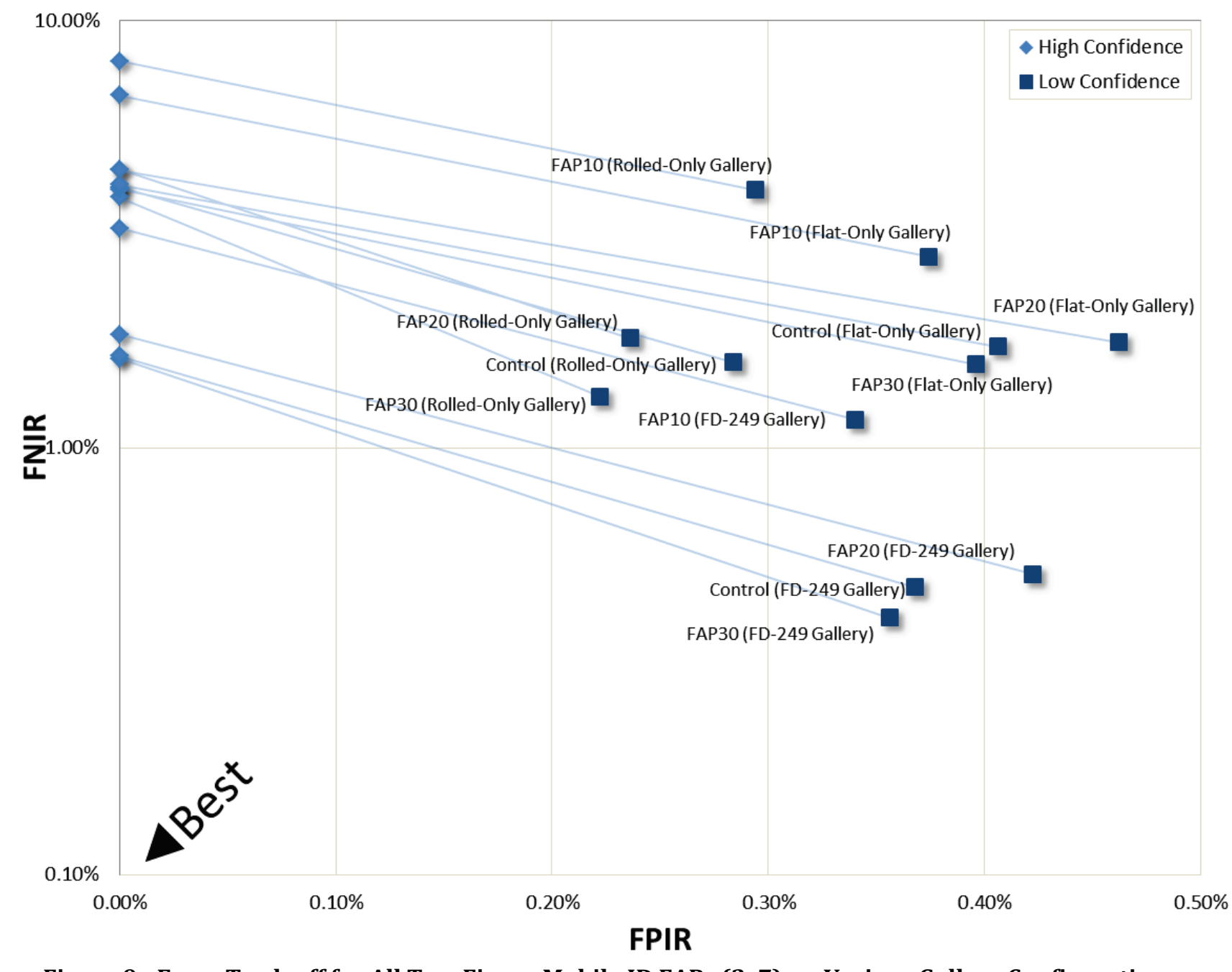

Figure 9 - Error Tradeoff for All Two Finger Mobile ID FAPs $(2,7)$ vs. Various Gallery Configurations 


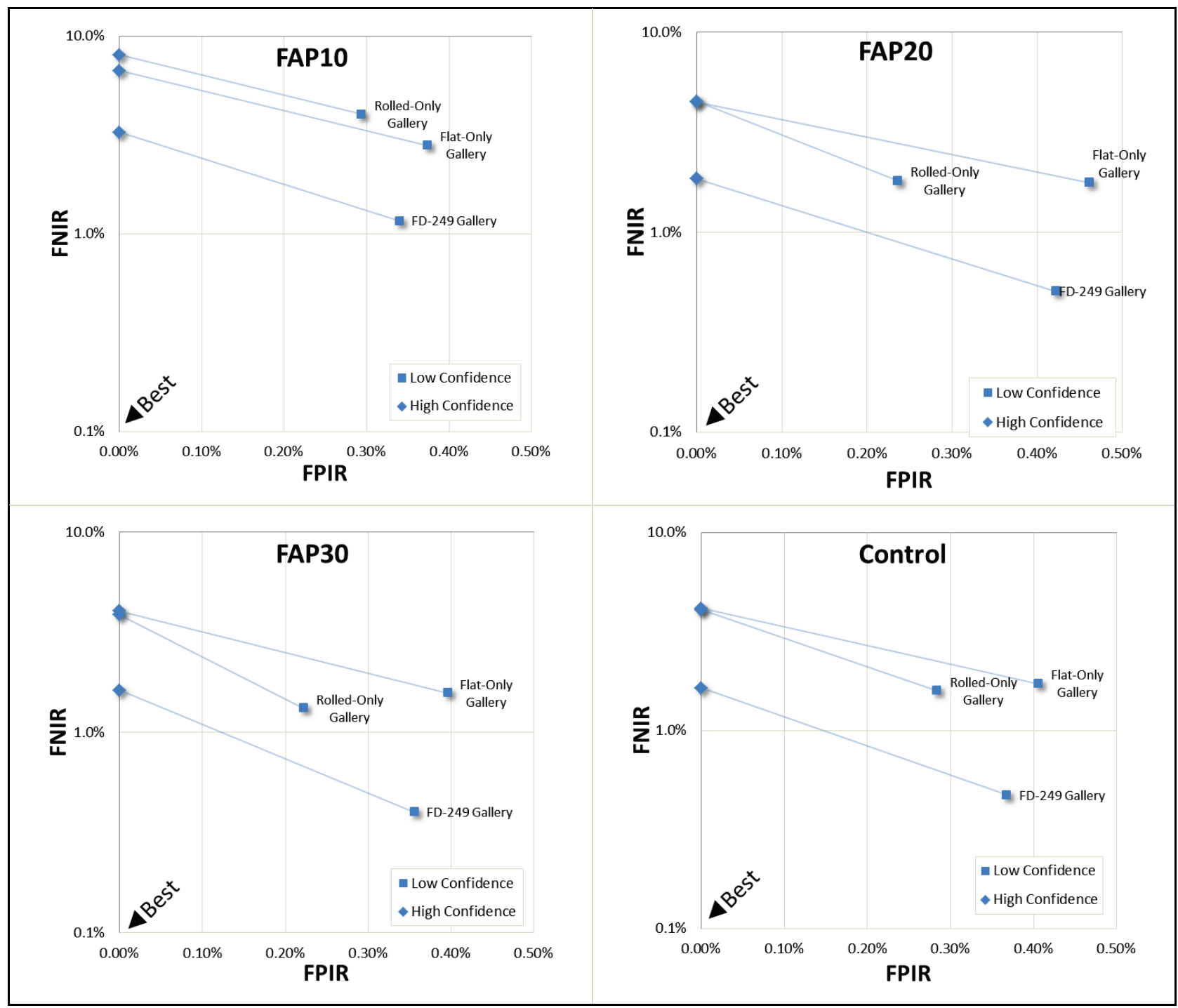

Figure 10 - Error Tradeoff for Each Two Finger Mobile ID FAP $(2,7)$ vs. Various Gallery Configurations

\subsubsection{Results for Objective 4}

For the two finger probe field scenario $(2,7)$ examined in this study objective, a full FD-249 set gallery emerges as the best gallery where all four FD-249 gallery test scenarios emerged at the top of the performance rankings (see Table 10), with FAP30 leading the pack at both low and high confidence thresholds (FNIR of $0.400 \%$ and $1.616 \%$ respectively). A rolled-only gallery favors optimization of FPIR for all Mobile ID FAPs at the low confidence threshold with the four lowest FPIR cases being the result of a rolled-only gallery configuration, and FAP30 again leading the pack with the lowest FPIR rate. At the high confidence system threshold, FPIR is flat at $0 \%$ for all cases. 


\subsection{Objective 5: System throughput behavior}

The next objective in this study was to measure the throughput of the system using various Mobile ID FAP scenarios using the full set FD-249 gallery. In addition to the various image-based scenarios, template-only based scenarios were also tested to provide a basis of throughput without the image processing overhead involved. The templates generated by the system were proprietary to the system and generated on the system environment prior to conducting the test. Finally, for the purpose of comparison, ten-print submissions using the system in both RISC and IDFP configuration were made and the throughput measured.

\subsubsection{Data for Objective 5}

Table 11 and Figure 11 provide median match times for the system processing of various Mobile ID FAP scenarios (image-only as well as template-only probe sets) using the full set FD-249 gallery, while Table 12 and Figure 12 provide median match times for system processing of various image-only Mobile ID FAP scenarios and finger combinations against the full set FD-249 gallery.

Table 11 - Median System Matching Times (milliseconds) for Selected FAPs vs. FD-249 Gallery

\begin{tabular}{|c|c|c|c|c|c|c|c|}
\hline Probe Type & & $\operatorname{FAP10}(2,7)$ & $\operatorname{FAP20}(2,7)$ & $\operatorname{FAP30}(2,7)$ & Control $(2,7)$ & Control (1-10), RISC & Control (1-10), IDFP \\
\hline \multirow[t]{2}{*}{ Image-only Probe } & Non-mated & 1966 & 2370 & 3059 & 2690 & 4336 & 3096 \\
\hline & Mated & 1841 & 2251 & 2964 & 2568 & 3362 & 3186 \\
\hline \multirow[t]{2}{*}{ Template-only Probe } & Non-mated & 763 & 807 & 936 & 851 & 2099 & 1918 \\
\hline & Mated & 663 & 707 & 870 & 756 & 1293 & 1739 \\
\hline
\end{tabular}

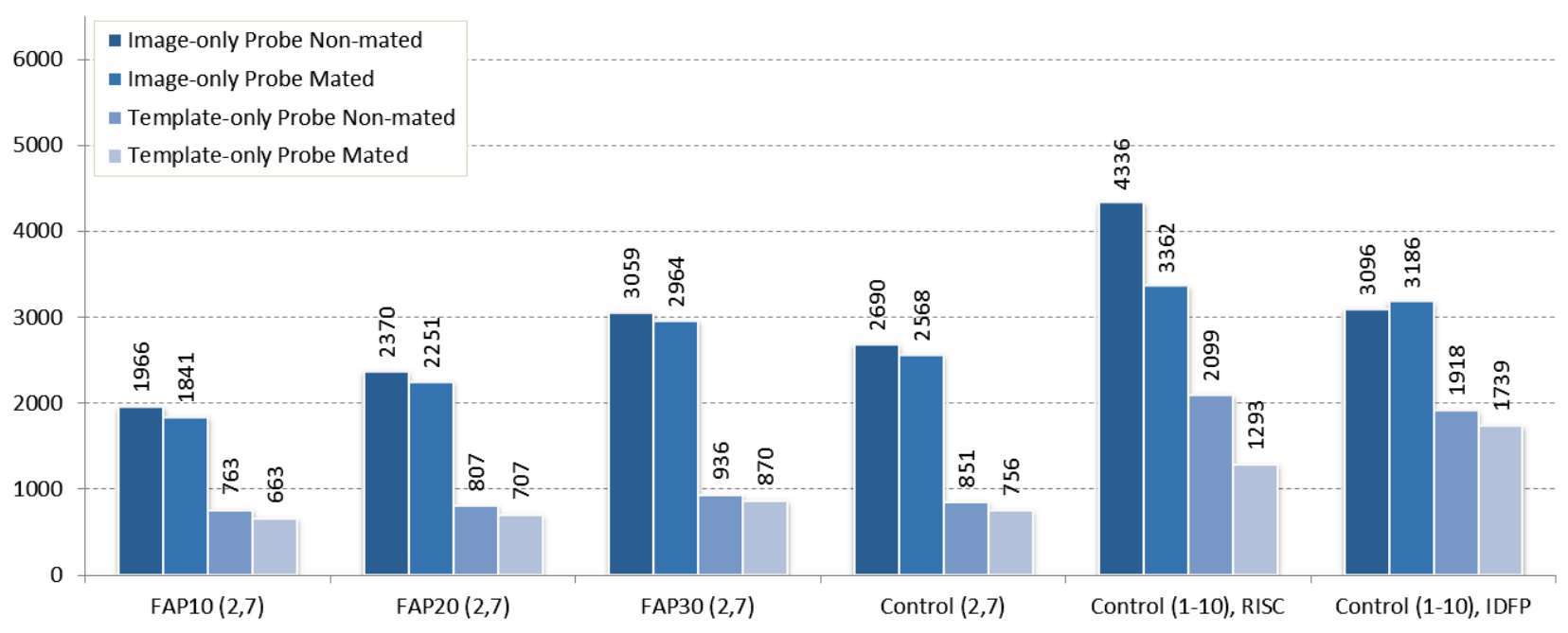

Figure 11 - Median Matching Times (milliseconds) for Selected FAPs vs. FD-249 Gallery 
Table 12 - Median Matching Times (milliseconds) for Image-Only Searches vs. Finger Combination

\begin{tabular}{|c|c|c|c|c|c|c|}
\hline \multirow[b]{2}{*}{ Probe Type } & & \multicolumn{5}{|c|}{ Finger Combination } \\
\hline & & 1,6 & 2,7 & $1,2,6,7$ & $2,3,7,8$ & $1-10$ \\
\hline \multirow[t]{2}{*}{ FAP10 } & Non-mated & 3068 & 1966 & 2155 & 2205 & 3377 \\
\hline & Mated & 2942 & 1841 & 1945 & 1996 & 2050 \\
\hline \multirow[t]{2}{*}{ FAP20 } & Non-mated & 3973 & 2370 & 2675 & 2664 & 3671 \\
\hline & Mated & 3928 & 2251 & 2505 & 2483 & 2537 \\
\hline \multirow[t]{2}{*}{ FAP30 } & Non-mated & 5323 & 3059 & 3713 & 3518 & 4400 \\
\hline & Mated & 5345 & 2964 & 3530 & 3327 & 3426 \\
\hline Control & Mated & 5467 & 2568 & 3481 & 2935 & 3362 \\
\hline
\end{tabular}

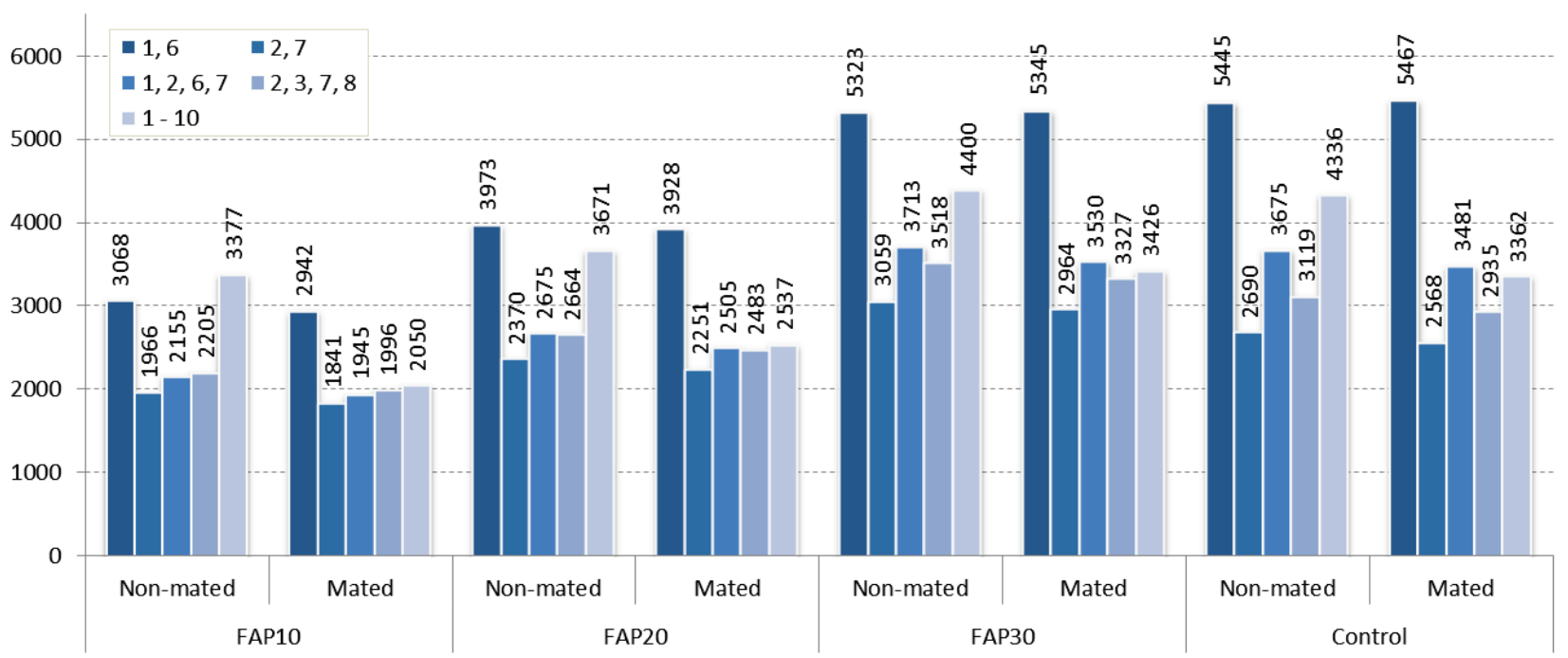

Figure 12 - Median Matching Times (milliseconds) for Image-Only Searches vs. Finger Combination 
Table 13 - Strictly Increasing FAP Median Match Times (FAP10 < FAP20 < FAP30) for Various Scenarios

\begin{tabular}{|c|c|c|c|c|c|c|c|}
\hline \multirow[b]{2}{*}{ Probe Contents } & \multirow[b]{2}{*}{ Probe Matching } & \multirow[b]{2}{*}{ Gallery } & \multicolumn{5}{|c|}{ Finger Combination Where Median Match Time for FAP10 < FAP20 < FAP30 } \\
\hline & & & 1,6 & 2,7 & $1,2,6,7$ & $2,3,7,8$ & $1-10$ \\
\hline Image & Non-mated & FD249 & TRUE & TRUE & TRUE & TRUE & TRUE \\
\hline Image & Non-mated & Flat & TRUE & TRUE & TRUE & TRUE & TRUE \\
\hline Image & Non-mated & Rolled & TRUE & TRUE & TRUE & TRUE & TRUE \\
\hline Image & Mated & FD249 & TRUE & TRUE & TRUE & TRUE & TRUE \\
\hline Image & Mated & Flat & TRUE & TRUE & TRUE & TRUE & TRUE \\
\hline Image & Mated & Rolled & TRUE & TRUE & TRUE & TRUE & TRUE \\
\hline Template & Non-mated & Flat & TRUE & TRUE & TRUE & TRUE & FALSE \\
\hline Template & Non-mated & Rolled & TRUE & TRUE & TRUE & TRUE & FALSE \\
\hline Template & Mated & Flat & TRUE & TRUE & TRUE & TRUE & FALSE \\
\hline Template & Mated & FD249 & TRUE & TRUE & TRUE & TRUE & FALSE \\
\hline Template & Mated & Rolled & TRUE & TRUE & TRUE & TRUE & FALSE \\
\hline
\end{tabular}

\subsubsection{Results for Objective 5}

System match times appear to be linked to image spatial geometry where median search time for all image searches FAPs strictly increases from FAP10 to FAP30. This strictly increasing pattern is also present in all template searches except for the ten-print template search cases where the FAP10 cases appear to incur a larger penalty than FAP20. The strictly increasing behavior is not consistent however between FAP30 and the Control case where FAP30 incurs a slight penalty versus the uncropped control case.

As noted before, the two thumbs $(1,6)$ case again appears to exhibit a significant penalty, with searches taking nearly twice as long as other finger combinations for a given FAP. 


\subsection{Objective 6: Identifying an Optimal Strategy}

The next objective in this study was to organize and rank all test cases together where data from all Mobile ID FAPs, matched against all gallery configurations and finger combinations was tabulated into a single data table (Table 14). This data was then assigned a rank based on the methodology described in section 3.8. This data allows for comparison of heterogeneous configurations (e.g., Mobile ID FAP10 using an FD-249 gallery vs. Mobile ID FAP30 using a flat-only gallery) at a glance.

Performance data (FPIR and FNIR) for each of the experimental treatments were each assigned a rank ranging from 1 (best) to 60 (worst) for each of FPIR and FNIR, at both low and high confidence threshold. The composite table of ranks and performance data is presented in Table $\mathbf{1 4}$ and an error trade-off graph is presented in Figure 13. 
4.6.1. Data for Objective 6

Table 14 - Performance Data Ranked by Distance-to-Optimal for Error Trade-off

\begin{tabular}{|c|c|c|c|c|c|c|c|c|c|c|c|c|}
\hline 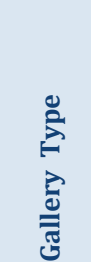 & 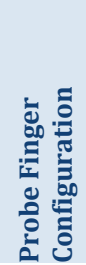 & 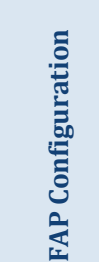 & 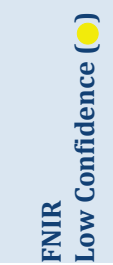 & 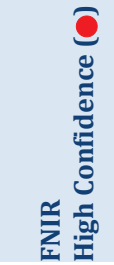 & 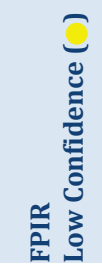 & 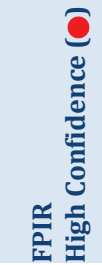 & 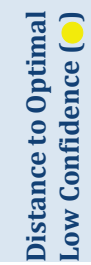 & 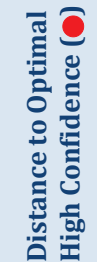 & 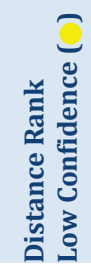 & 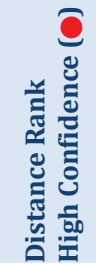 & 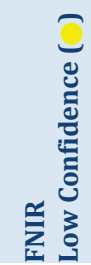 & 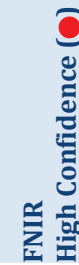 \\
\hline FD-249 & $1-10$ & FAP30 & $0.098 \%$ & $0.206 \%$ & $0.054 \%$ & $0.000 \%$ & 0.0011 & 0.0021 & 1 & 1 & L1 & $\mathrm{H} 1$ \\
\hline FD-249 & $1-10$ & Control & $0.134 \%$ & $0.218 \%$ & $0.052 \%$ & $0.000 \%$ & 0.0014 & 0.0022 & 2 & 2 & L2 & $\mathrm{H} 2$ \\
\hline FD-249 & $1-10$ & FAP20 & $0.190 \%$ & $0.306 \%$ & $0.060 \%$ & $0.000 \%$ & 0.0020 & 0.0031 & 3 & 3 & L3 & H3 \\
\hline FD-249 & $2,3,7,8$ & FAP30 & $0.160 \%$ & $0.338 \%$ & $0.548 \%$ & $0.000 \%$ & 0.0057 & 0.0034 & 10 & 4 & L10 & $\mathrm{H} 4$ \\
\hline FD-249 & $2,3,7,8$ & Control & $0.192 \%$ & $0.374 \%$ & $0.546 \%$ & $0.000 \%$ & 0.0058 & 0.0037 & 11 & 5 & L11 & H5 \\
\hline FD-249 & $1,2,6,7$ & FAP30 & $0.216 \%$ & $0.390 \%$ & $0.122 \%$ & $0.000 \%$ & 0.0025 & 0.0039 & 4 & 6 & L4 & H6 \\
\hline FD-249 & $1,2,6,7$ & Control & $0.226 \%$ & $0.410 \%$ & $0.158 \%$ & $0.000 \%$ & 0.0028 & 0.0041 & 5 & 7 & L5 & H7 \\
\hline FD-249 & $2,3,7,8$ & FAP20 & $0.236 \%$ & $0.442 \%$ & $0.650 \%$ & $0.000 \%$ & 0.0069 & 0.0044 & 16 & 8 & L16 & H8 \\
\hline Flat & $1-10$ & FAP30 & $0.538 \%$ & $0.662 \%$ & $0.060 \%$ & $0.000 \%$ & 0.0054 & 0.0066 & 8 & 9 & L8 & H9 \\
\hline FD-249 & $1,2,6,7$ & FAP20 & $0.464 \%$ & $0.720 \%$ & $0.128 \%$ & $0.000 \%$ & 0.0048 & 0.0072 & 6 & 10 & L6 & H10 \\
\hline Rolled & $1-10$ & FAP30 & $0.564 \%$ & $0.768 \%$ & $0.062 \%$ & $0.000 \%$ & 0.0057 & 0.0077 & 9 & 11 & L9 & H11 \\
\hline Flat & $1-10$ & Control & $0.686 \%$ & $0.786 \%$ & $0.066 \%$ & $0.000 \%$ & 0.0069 & 0.0079 & 15 & 12 & L15 & H12 \\
\hline Rolled & $1-10$ & Control & $0.688 \%$ & $0.868 \%$ & $0.036 \%$ & $0.000 \%$ & 0.0069 & 0.0087 & 14 & 13 & L14 & H13 \\
\hline Rolled & $2,3,7,8$ & FAP30 & $0.652 \%$ & $1.010 \%$ & $0.258 \%$ & $0.000 \%$ & 0.0070 & 0.0101 & 17 & 14 & L17 & H14 \\
\hline Flat & $1-10$ & FAP20 & $0.946 \%$ & $1.084 \%$ & $0.052 \%$ & $0.000 \%$ & 0.0095 & 0.0108 & 21 & 15 & L21 & H15 \\
\hline Flat & $1,2,6,7$ & FAP30 & $0.796 \%$ & $1.094 \%$ & $0.138 \%$ & $0.000 \%$ & 0.0081 & 0.0109 & 18 & 16 & L18 & H16 \\
\hline Flat & $2,3,7,8$ & FAP30 & $0.714 \%$ & $1.124 \%$ & $0.714 \%$ & $0.000 \%$ & 0.0101 & 0.0112 & 23 & 17 & L23 & H17 \\
\hline Flat & $1,2,6,7$ & Control & $0.888 \%$ & $1.188 \%$ & $0.158 \%$ & $0.000 \%$ & 0.0090 & 0.0119 & 19 & 18 & L19 & H18 \\
\hline Rolled & $2,3,7,8$ & Control & $0.892 \%$ & $1.194 \%$ & $0.344 \%$ & $0.000 \%$ & 0.0096 & 0.0119 & 22 & 19 & L22 & H19 \\
\hline Flat & $2,3,7,8$ & Control & $0.848 \%$ & $1.214 \%$ & $0.644 \%$ & $0.000 \%$ & 0.0106 & 0.0121 & 25 & 20 & L25 & $\mathrm{H} 20$ \\
\hline Rolled & $1-10$ & FAP20 & $1.030 \%$ & $1.230 \%$ & $0.028 \%$ & $0.000 \%$ & 0.0103 & 0.0123 & 24 & 21 & L24 & H 21 \\
\hline Rolled & $1,2,6,7$ & FAP30 & $0.904 \%$ & $1.234 \%$ & $0.098 \%$ & $0.000 \%$ & 0.0091 & 0.0123 & 20 & 22 & L20 & H 22 \\
\hline Flat & $2,3,7,8$ & FAP20 & $0.936 \%$ & $1.342 \%$ & $0.664 \%$ & $0.000 \%$ & 0.0115 & 0.0134 & 28 & 23 & L28 & H 23 \\
\hline Rolled & $2,3,7,8$ & FAP20 & $1.016 \%$ & $1.390 \%$ & $0.356 \%$ & $0.000 \%$ & 0.0108 & 0.0139 & 26 & 24 & L26 & $\mathrm{H} 24$ \\
\hline Rolled & $1,2,6,7$ & Control & $1.114 \%$ & $1.420 \%$ & $0.108 \%$ & $0.000 \%$ & 0.0112 & 0.0142 & 27 & 25 & L27 & H25 \\
\hline FD-249 & 2,7 & FAP30 & $0.400 \%$ & $1.616 \%$ & $0.356 \%$ & $0.000 \%$ & 0.0054 & 0.0162 & 7 & 26 & L7 & H26 \\
\hline FD-249 & 2,7 & Control & $0.472 \%$ & $1.640 \%$ & $0.368 \%$ & $0.000 \%$ & 0.0060 & 0.0164 & 12 & 27 & L12 & H27 \\
\hline FD-249 & $2,3,7,8$ & FAP10 & $1.372 \%$ & $1.714 \%$ & $0.622 \%$ & $0.000 \%$ & 0.0151 & 0.0171 & 32 & 28 & L32 & H 28 \\
\hline Flat & $1,2,6,7$ & FAP20 & $1.364 \%$ & $1.734 \%$ & $0.166 \%$ & $0.000 \%$ & 0.0137 & 0.0173 & 31 & 29 & L31 & H 29 \\
\hline FD-249 & 2,7 & FAP20 & $0.506 \%$ & $1.844 \%$ & $0.422 \%$ & $0.000 \%$ & 0.0066 & 0.0184 & 13 & 30 & L13 & H30 \\
\hline Rolled & $1,2,6,7$ & FAP20 & $1.838 \%$ & $2.238 \%$ & $0.104 \%$ & $0.000 \%$ & 0.0184 & 0.0224 & 38 & 31 & L38 & H31 \\
\hline Flat & $2,3,7,8$ & FAP10 & $2.660 \%$ & $3.260 \%$ & $0.704 \%$ & $0.000 \%$ & 0.0275 & 0.0326 & 42 & 32 & L42 & H32 \\
\hline FD-249 & 2,7 & FAP10 & $1.164 \%$ & $3.272 \%$ & $0.340 \%$ & $0.000 \%$ & 0.0121 & 0.0327 & 29 & 33 & L29 & H33 \\
\hline FD-249 & 1,6 & Control & $1.890 \%$ & $3.560 \%$ & $0.046 \%$ & $0.000 \%$ & 0.0189 & 0.0356 & 39 & 34 & L39 & H34 \\
\hline FD-249 & 1,6 & FAP30 & $1.964 \%$ & $3.598 \%$ & $0.090 \%$ & $0.000 \%$ & 0.0197 & 0.0360 & 40 & 35 & L40 & H35 \\
\hline Rolled & 2,7 & FAP30 & $1.318 \%$ & $3.862 \%$ & $0.222 \%$ & $0.000 \%$ & 0.0134 & 0.0386 & 30 & 36 & L30 & H36 \\
\hline FD-249 & $1-10$ & FAP10 & $3.740 \%$ & $3.940 \%$ & $0.034 \%$ & $0.000 \%$ & 0.0374 & 0.0394 & 45 & 37 & L45 & H37 \\
\hline Flat & 2,7 & FAP30 & $1.570 \%$ & $4.032 \%$ & $0.396 \%$ & $0.000 \%$ & 0.0162 & 0.0403 & 34 & 38 & L34 & H38 \\
\hline FD-249 & $1,2,6,7$ & FAP10 & $3.658 \%$ & $4.040 \%$ & $0.146 \%$ & $0.000 \%$ & 0.0366 & 0.0404 & 44 & 39 & L44 & H39 \\
\hline Rolled & 2,7 & Control & $1.588 \%$ & $4.078 \%$ & $0.284 \%$ & $0.000 \%$ & 0.0161 & 0.0408 & 33 & 40 & L33 & $\mathrm{H} 40$ \\
\hline Flat & 2,7 & Control & $1.726 \%$ & $4.138 \%$ & $0.406 \%$ & $0.000 \%$ & 0.0177 & 0.0414 & 35 & 41 & L35 & H41 \\
\hline Flat & 2,7 & FAP20 & $1.766 \%$ & $4.474 \%$ & $0.462 \%$ & $0.000 \%$ & 0.0183 & 0.0447 & 37 & 42 & L37 & $\mathrm{H} 42$ \\
\hline Rolled & 2,7 & FAP20 & $1.806 \%$ & $4.494 \%$ & $0.236 \%$ & $0.000 \%$ & 0.0182 & 0.0449 & 36 & 43 & L36 & $\mathrm{H} 43$ \\
\hline Rolled & $2,3,7,8$ & FAP10 & $4.212 \%$ & $4.768 \%$ & $0.462 \%$ & $0.000 \%$ & 0.0424 & 0.0477 & 49 & 44 & L49 & H44 \\
\hline FD-249 & 1,6 & FAP20 & $2.620 \%$ & $5.130 \%$ & $0.106 \%$ & $0.000 \%$ & 0.0262 & 0.0513 & 41 & 45 & L41 & $\mathrm{H} 45$ \\
\hline Flat & 1,6 & Control & $3.846 \%$ & $6.032 \%$ & $0.064 \%$ & $0.000 \%$ & 0.0385 & 0.0603 & 46 & 46 & L46 & H46 \\
\hline Flat & 1,6 & FAP30 & $3.930 \%$ & $6.124 \%$ & $0.082 \%$ & $0.000 \%$ & 0.0393 & 0.0612 & 47 & 47 & L47 & $\mathrm{H} 47$ \\
\hline Flat & $1,2,6,7$ & FAP10 & $5.786 \%$ & $6.334 \%$ & $0.182 \%$ & $0.000 \%$ & 0.0579 & 0.0633 & 53 & 48 & L53 & $\mathrm{H} 48$ \\
\hline Flat & 2,7 & FAP10 & $2.806 \%$ & $6.694 \%$ & $0.374 \%$ & $0.000 \%$ & 0.0283 & 0.0669 & 43 & 49 & L43 & H49 \\
\hline Flat & $1-10$ & FAP10 & $6.438 \%$ & $6.706 \%$ & $0.036 \%$ & $0.000 \%$ & 0.0644 & 0.0671 & 56 & 50 & L56 & $\mathrm{H} 50$ \\
\hline Rolled & 1,6 & Control & $4.298 \%$ & $7.560 \%$ & $0.054 \%$ & $0.000 \%$ & 0.0430 & 0.0756 & 50 & 51 & L50 & H51 \\
\hline Rolled & 1,6 & FAP30 & $4.380 \%$ & $7.630 \%$ & $0.076 \%$ & $0.000 \%$ & 0.0438 & 0.0763 & 51 & 52 & L51 & H52 \\
\hline Rolled & $1-10$ & FAP10 & $7.539 \%$ & $7.899 \%$ & $0.064 \%$ & $0.000 \%$ & 0.0754 & 0.0790 & 57 & 53 & L57 & H53 \\
\hline Rolled & 2,7 & FAP10 & $4.024 \%$ & $8.048 \%$ & $0.294 \%$ & $0.000 \%$ & 0.0403 & 0.0805 & 48 & 54 & L48 & H54 \\
\hline Rolled & $1,2,6,7$ & FAP10 & $7.704 \%$ & $8.372 \%$ & $0.164 \%$ & $0.000 \%$ & 0.0771 & 0.0837 & 58 & 55 & L58 & H55 \\
\hline Flat & 1,6 & FAP20 & $5.268 \%$ & $8.504 \%$ & $0.132 \%$ & $0.000 \%$ & 0.0527 & 0.0850 & 52 & 56 & L52 & H56 \\
\hline FD-249 & 1,6 & FAP10 & $5.938 \%$ & $10.402 \%$ & $0.208 \%$ & $0.000 \%$ & 0.0594 & 0.1040 & 55 & 57 & L55 & H57 \\
\hline Rolled & 1,6 & FAP20 & $5.860 \%$ & $10.850 \%$ & $0.080 \%$ & $0.000 \%$ & 0.0586 & 0.1085 & 54 & 58 & L54 & H58 \\
\hline Flat & 1,6 & FAP10 & $11.122 \%$ & $16.350 \%$ & $0.218 \%$ & $0.000 \%$ & 0.1112 & 0.1635 & 60 & 59 & L60 & H59 \\
\hline Rolled & 1,6 & FAP10 & $10.610 \%$ & $18.566 \%$ & $0.176 \%$ & $0.000 \%$ & 0.1061 & 0.1857 & 59 & 60 & L59 & $\mathrm{H} 60$ \\
\hline
\end{tabular}




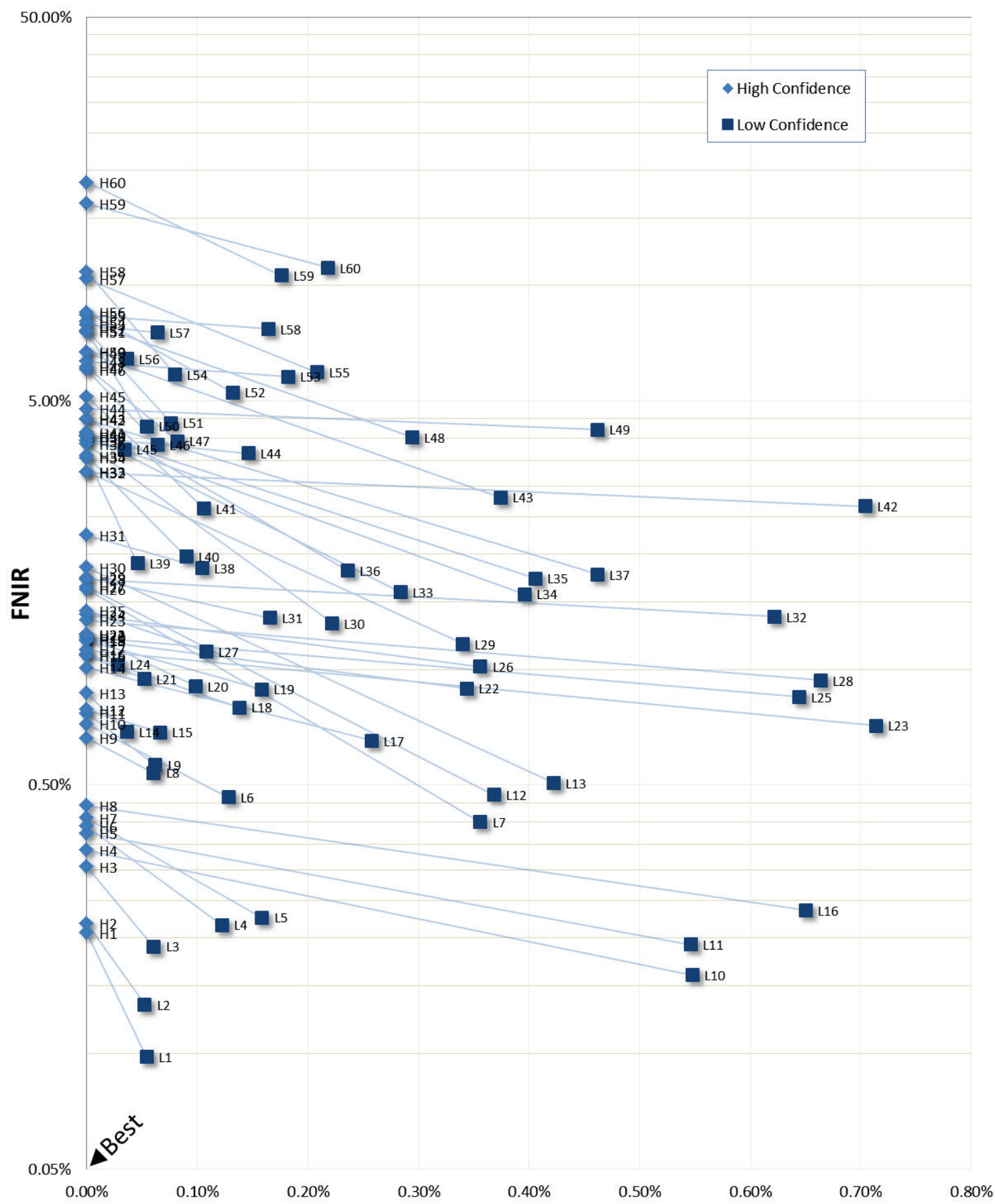

FPIR

Figure 13 - Error Tradeoff for all Test Cases ${ }^{9}$

\footnotetext{
${ }^{9}$ See Table 14 for legend key
} 


\subsubsection{Results for Objective 6}

Using the data in Table 14/Figure 13 spanning all 60 cases examined in this study, the case of FAP30 two finger $(2,7)$ using the FD-249 full set gallery emerges as the best two finger FAP for the high confidence system threshold in terms of error trade-off with a distance-to-optimal of 0.0162 . The highest ranking FAP10 case when using an FD-249 database is that of four finger $(2,3,7,8)$ FAP10 with a distance-to-origin of 0.0171 ranking it higher than FAP20 two finger $(2,7)$ but lower than both two finger $(2,7)$ FAP30 and two finger $(2,7)$ control cases. Given this information, in cases where legacy FAP10 hardware needs to be used, FAP10 $(2,3,7,8)$ with $\mathrm{d}=0.0171$ may be a competitive alternative in terms of error trade-off with the top ranking case of FAP30 two finger $(2,7)$ with $d=0.0162$.

Looking from the bottom end of the performance rankings, the two finger $(1,6)$ occupies the lowest rankings with all gallery configurations, with the two finger $(1,6)$ FAP10 case coming in dead last among all 60 cases examined for the high confidence system threshold. 


\subsection{Objective 7: Exploratory Examination of Emerging Small-Platen Capture Devices}

As mobile/handheld information devices mature and evolve, the market has seen a gradual proliferation of biometric technology in these devices primarily for access control either as a replacement for or as an augmentation of traditional password and/or gesture based security measures. These biometric technologies include a new generation of very-small platen devices such as the fingerprint sensor embedded in the iPhone ${ }^{10}$ $5 \mathrm{~s}$ (see Figure 14). While such sensors are intended for verification purposes (also referred to as one to one, or $1: 1)$ and the usage of these very-small sensors for one-to-many searching $(1: \mathrm{N})$ has not been endorsed by any vendors, this study included an exploratory examination on the use of such a small platen device in a one-tomany biometric-search scenario $(1: \mathrm{N})$. This was done by creating a $1 / 4{ }^{\prime \prime}(6.35 \mathrm{~mm})$ square fingerprint image and testing that image using the same protocols that other Mobile ID FAPs were tested. The gallery used for this test was populated with full FD-249 image sets and the system was configured with RISC system optimization.

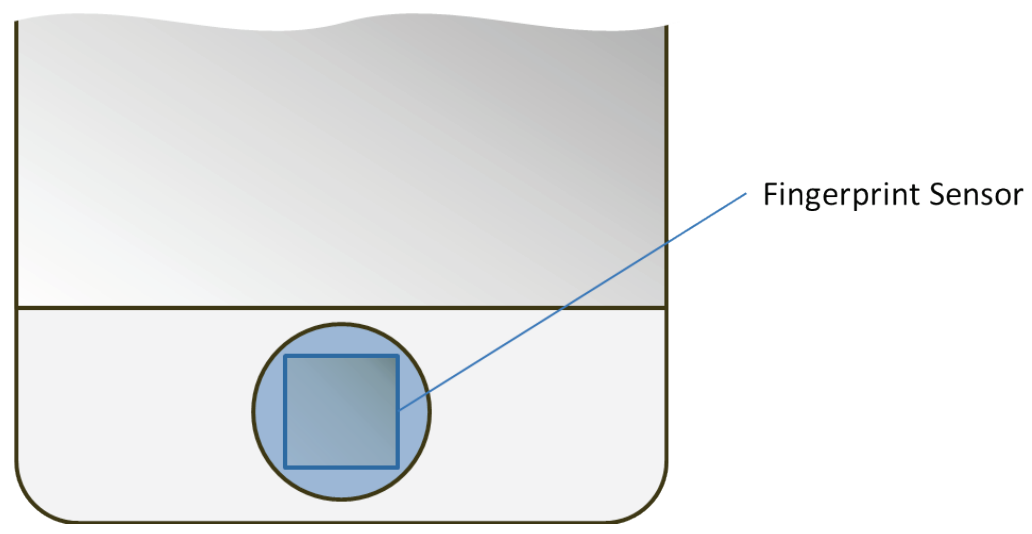

Figure 14 - Example of an Embedded Small Biometric Verification Sensor Such as the iPhone ${ }^{10} 5 \mathrm{~S}$

\subsubsection{Data for Objective 7}

Figure 15 below shows summary data from tests utilizing various finger combination for all Mobile ID FAPs described in Table 1 plus the experimental 1/4" FAP data. Table 14 summarizes the data in Figure 15, and ranks the values from 1 (best) to 25 (worst) for each of the performance metrics (FPIR and FNIR) and both system confidence thresholds (High and Low) using the distance-to-origin methodology described in section 3.8.

10 iPhone is a registered trademark of Apple Inc., registered in the U.S. and other countries. 
Table 15 - Comparison of Experimental 1/4" FAP Versus Traditional Mobile ID FAPs

\begin{tabular}{|c|c|c|c|c|c|c|c|c|c|c|}
\hline 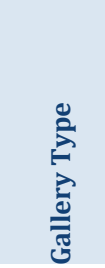 & 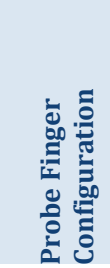 & 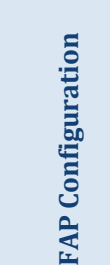 & 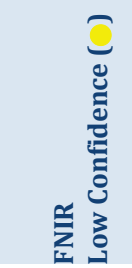 & 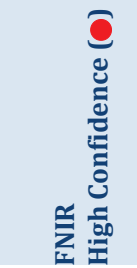 & 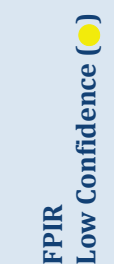 & 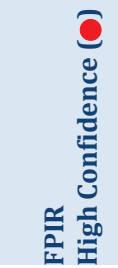 & 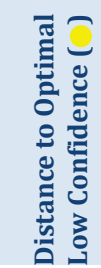 & 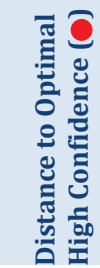 & 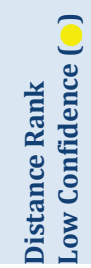 & 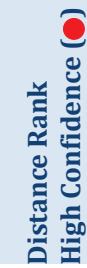 \\
\hline FD-249 & $1-10$ & FAP30 & $0.098 \%$ & $0.206 \%$ & $0.054 \%$ & $0.000 \%$ & 0.0011 & 0.0021 & 1 & 1 \\
\hline FD-249 & $1-10$ & Control & $0.134 \%$ & $0.218 \%$ & $0.052 \%$ & $0.000 \%$ & 0.0014 & 0.0022 & 2 & 2 \\
\hline FD-249 & $1-10$ & FAP20 & $0.190 \%$ & $0.306 \%$ & $0.060 \%$ & $0.000 \%$ & 0.0020 & 0.0031 & 3 & 3 \\
\hline FD-249 & $2,3,7,8$ & FAP30 & $0.160 \%$ & $0.338 \%$ & $0.548 \%$ & $0.000 \%$ & 0.0057 & 0.0034 & 8 & 4 \\
\hline FD-249 & $2,3,7,8$ & Control & $0.192 \%$ & $0.374 \%$ & $0.546 \%$ & $0.000 \%$ & 0.0058 & 0.0037 & 9 & 5 \\
\hline FD-249 & $1,2,6,7$ & FAP30 & $0.216 \%$ & $0.390 \%$ & $0.122 \%$ & $0.000 \%$ & 0.0025 & 0.0039 & 4 & 6 \\
\hline FD-249 & $1,2,6,7$ & Control & $0.226 \%$ & $0.410 \%$ & $0.158 \%$ & $0.000 \%$ & 0.0028 & 0.0041 & 5 & 7 \\
\hline FD-249 & $2,3,7,8$ & FAP20 & $0.236 \%$ & $0.442 \%$ & $0.650 \%$ & $0.000 \%$ & 0.0069 & 0.0044 & 12 & 8 \\
\hline FD-249 & $1,2,6,7$ & FAP20 & $0.464 \%$ & $0.720 \%$ & $0.128 \%$ & $0.000 \%$ & 0.0048 & 0.0072 & 6 & 9 \\
\hline FD-249 & 2,7 & FAP30 & $0.400 \%$ & $1.616 \%$ & $0.356 \%$ & $0.000 \%$ & 0.0054 & 0.0162 & 7 & 10 \\
\hline FD-249 & 2,7 & Control & $0.472 \%$ & $1.640 \%$ & $0.368 \%$ & $0.000 \%$ & 0.0060 & 0.0164 & 10 & 11 \\
\hline FD-249 & $2,3,7,8$ & FAP10 & $1.372 \%$ & $1.714 \%$ & $0.622 \%$ & $0.000 \%$ & 0.0151 & 0.0171 & 14 & 12 \\
\hline FD-249 & 2,7 & FAP20 & $0.506 \%$ & $1.844 \%$ & $0.422 \%$ & $0.000 \%$ & 0.0066 & 0.0184 & 11 & 13 \\
\hline FD-249 & 2,7 & FAP10 & $1.164 \%$ & $3.272 \%$ & $0.340 \%$ & $0.000 \%$ & 0.0121 & 0.0327 & 13 & 14 \\
\hline FD-249 & 1,6 & Control & $1.890 \%$ & $3.560 \%$ & $0.046 \%$ & $0.000 \%$ & 0.0189 & 0.0356 & 15 & 15 \\
\hline FD-249 & 1,6 & FAP30 & $1.964 \%$ & $3.598 \%$ & $0.090 \%$ & $0.000 \%$ & 0.0197 & 0.0360 & 16 & 16 \\
\hline FD-249 & $1-10$ & FAP10 & $3.740 \%$ & $3.940 \%$ & $0.034 \%$ & $0.000 \%$ & 0.0374 & 0.0394 & 19 & 17 \\
\hline FD-249 & $1,2,6,7$ & FAP10 & $3.658 \%$ & $4.040 \%$ & $0.146 \%$ & $0.000 \%$ & 0.0366 & 0.0404 & 18 & 18 \\
\hline FD-249 & 1,6 & FAP20 & $2.620 \%$ & $5.130 \%$ & $0.106 \%$ & $0.000 \%$ & 0.0262 & 0.0513 & 17 & 19 \\
\hline FD-249 & 1,6 & FAP10 & $5.938 \%$ & $10.402 \%$ & $0.208 \%$ & $0.000 \%$ & 0.0594 & 0.1040 & 20 & 20 \\
\hline FD-249 & $1-10$ & $1 / 4 "$ FAP & $20.122 \%$ & $47.432 \%$ & $0.000 \%$ & $0.000 \%$ & 0.2012 & 0.4743 & 21 & 21 \\
\hline FD-249 & $2,3,7,8$ & $1 / 4 "$ FAP & $31.558 \%$ & $64.524 \%$ & $0.000 \%$ & $0.000 \%$ & 0.3156 & 0.6452 & 22 & 22 \\
\hline FD-249 & $1,2,6,7$ & $1 / 4 "$ FAP & $33.214 \%$ & $69.905 \%$ & $0.000 \%$ & $0.000 \%$ & 0.3321 & 0.6991 & 23 & 23 \\
\hline FD-249 & 2,7 & 1/4" FAP & $54.894 \%$ & $94.440 \%$ & $0.002 \%$ & $0.000 \%$ & 0.5489 & 0.9444 & 24 & 24 \\
\hline FD-249 & 1,6 & 1/4" FAP & $66.687 \%$ & $97.380 \%$ & $0.002 \%$ & $0.000 \%$ & 0.6669 & 0.9738 & 25 & 25 \\
\hline
\end{tabular}

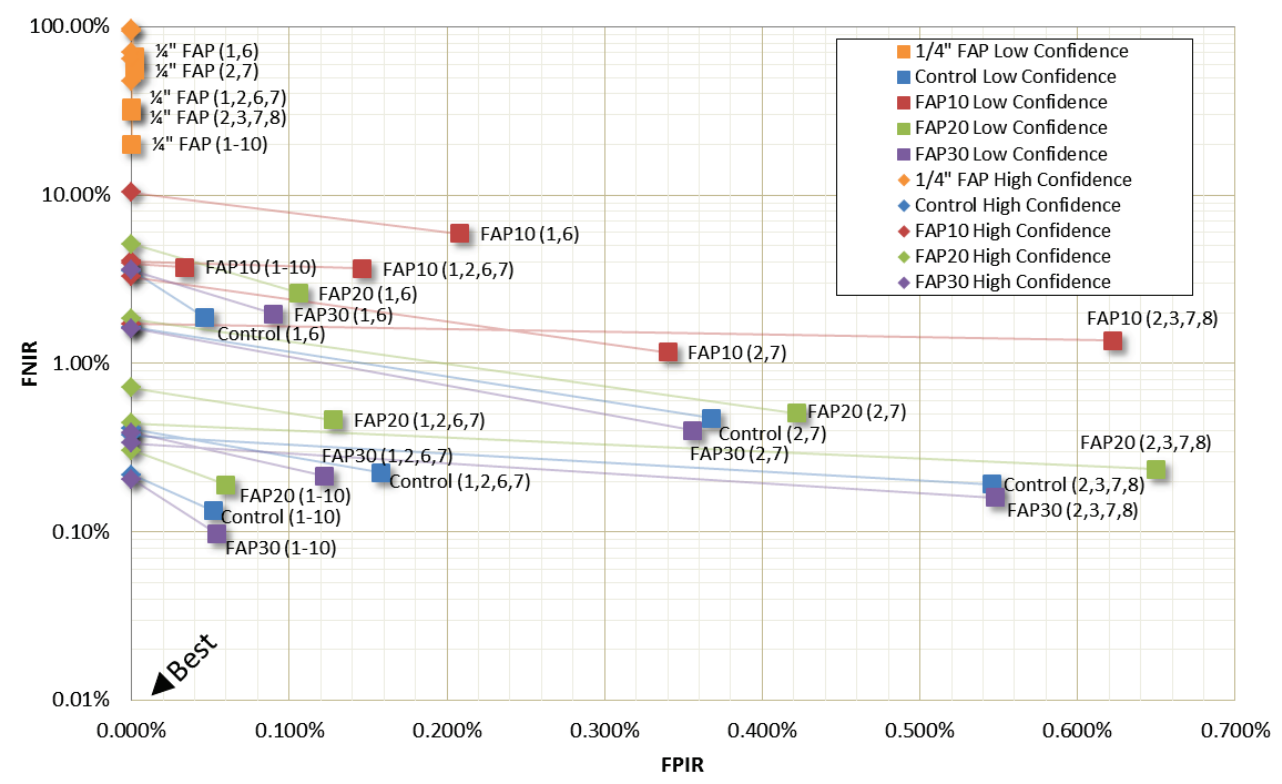

Figure 15 - Error Tradeoff Comparison of Experimental 1/4" FAP Versus Traditional Mobile ID FAPs 


\subsubsection{Results for Objective 7}

The smaller platen sensors present a significant advance in the area of authentication and verification; their application in one-to-many $(1: \mathrm{N})$ searching may be premature given the results from this exploratory investigation. While the FPIR rates remain reasonable and on a par with traditional Mobile ID FAPs examined in this study, FNIR takes a significant performance hit when using these images. In the worst case scenario, at the high confidence system threshold, FNIR peaks at $97.380 \%$ for the case of the two finger $(1,6)$ combination using the experimental 1/4" FAP. In the best case scenario where all ten fingers can be captured, the experimental $1 / 4$ " FAP yields an FNIR of $47.432 \%$, with an FPIR of $0.0 \%$. 


\section{Discussion}

Examining various two finger $(2,7)$ Mobile ID FAP scenarios with a gallery of full FD-249 fingerprint sets (section 4.2.1) shows that FAP10 is at a disadvantage when compared to other FAPs. In terms of FNIR, FAP10 submissions fail to identify their target twice as often as FAP20 (1.164\% vs. $0.506 \%)$ at the system low confidence threshold. For the high confidence threshold, FNIR for FAP10 is almost twice that of FAP20 (3.270\% vs. $1.844 \%$ ). Error tradeoff analysis using distance-to-optimal calculations (section 3.8) places the FAP10 case as last-ranked and worst for both low and high confidence FNIR, while FAP30 is best for both thresholds. A surprise finding was that the FAP30 case outperformed the control case, though by a very small margin. While this performance difference is small (e.g., an FNIR of $1.616 \%$ vs $1.640 \%$ for the two finger $(2,7)$ case at the high confidence threshold) this difference is statistically significant ( $\mathrm{p}<0.0001$ level, using Wilcoxon signed-rank test) and this pattern was observed in several other case clusters where FAP30 outperformed the control case ever so slightly.

For the two finger $(2,7)$ Mobile ID FAP scenarios with a full FD-249 gallery shows that FPIR rates for all cases is flat at $0 \%$ at the high confidence threshold, and none of the Mobile ID FAPs show any disadvantage over the others in the high confidence scenario. For the low confidence system threshold scenario, the difference in FPIR is very small with FAP10 showing a very negligible advantage over FAP30 ( $0.340 \%$ vs. $0.356 \%)$.

Examining cases of various finger combinations versus a full FD-249 gallery (section 4.3.1) shows that the FAP30 (1-10) finger case excels all others, followed by the Control (1-10) finger case, and then the FAP20 (1-10) finger case. This ranking is true for both the high and low confidence thresholds. On the other end of the scale, the FAP10 $(1,6)$ two finger case ranks last in performance for both the high and low confidence thresholds.

As with the two finger $(2,7)$ cases, all finger combinations at the high confidence system threshold yielded FPIR performance that was flat at $0 \%$ for all cases and appeared not to be impacted by the various finger combination treatments. At the low confidence system threshold, FPIR does show some fluctuation but the fluctuation is generally small with all values below $1 \%$.

FNIR performance is widely impacted by the number of fingers in the search probe and FNIR performance favors cases where the search probe had more finger impressions (e.g., 10 finger cases). FNIR at the high confidence system threshold ranges from $0.206 \%$ for the FAP30 (1-10) finger combination treatment, to $10.402 \%$ for the FAP10 $(1,6)$ finger combination treatment. This effect is so marked in that FNIR for the high confidence threshold 9 out of 10 cases in the top half of the ranked performance were either 4 or ten finger cases. FNIR performance at the low confidence threshold nearly mimics the high confidence performance, with the FNIR rates better than halved (i.e., $0.098 \%$ vs. $0.206 \%$ ) for the (1-10) FAP30 case which emerged as the best.

In examining the impact of gallery configuration on system performance (section 4.4.1), various Mobile ID FAPs in two finger $(2,7)$ configuration were tested against several possible gallery configurations (Table 4). For all Mobile ID scenarios examined, a full FD-249 set gallery emerged as the best gallery where all four FD-249 test scenarios were at the top of the performance rankings (see Table 10) using the distance based performance methodology (section 3.8), with FAP30 leading the pack at both low and high confidence thresholds (FNIR of $0.400 \%$ and $1.616 \%$ respectively). A rolled-only gallery favors optimization of FPIR for all Mobile ID FAPs at the low confidence threshold with the four lowest FPIR cases being the result of a rolled-only gallery configuration, and FAP30 again leading the pack with the lowest FPIR rate. At the high confidence system threshold, FPIR is flat at $0 \%$ for all cases.

Comparing the various Mobile ID FAP scenarios with a two finger $(2,7)$ treatment and a gallery consisting of various configurations of the system for impact on system throughput (section 4.5.1) shows that the system match times appear closely linked to image spatial geometry where median search times for all image search FAPs consistently increases from FAP10 to FAP30. This consistently increasing pattern is also present in all template searches and therefore not purely linked to image processing times. An exception to this increasing 
pattern is the ten-print template search cases where the FAP10 cases appear to incur a larger penalty than FAP20, and between FAP30 and the Control case where FAP30 incurs a slight penalty versus the uncropped control case. It should be emphasized however that the template-only matching scenarios do not suggest the elimination of the template generation processes and were included in this study to demonstrate the effect of the smaller Mobile ID templates on system throughput behavior without the overhead of image processing. In normal operation, template generation processes must ultimately occur somewhere in the processing workflow in order to conduct a template-only match.

Utilizing a rank based strategy based on distance-from optimal/origin (section 4.6.1), the absolute best ranked performance case is that of Mobile ID FAP30 (1-10) when matched against a full FD-249 gallery for both the low and high confidence thresholds. The case of FAP30 two finger $(2,7)$ using the FD-249 full set gallery emerges as the best two finger FAP for the high confidence system threshold in terms of error trade-off with a distance-tooptimal of 0.0162 . The highest ranking FAP10 case using an FD-249 database is that of four finger $(2,3,7,8)$ with a distance of 0.0171 ranking it higher than FAP20 two finger $(2,7)$ but lower than both two finger FAP30 and two finger control cases. Given this information, in cases where legacy FAP10 hardware needs to be used, FAP10 four finger $(2,3,7,8)$ may be a competitive alternative in terms of error trade-off with the top ranking case of FAP30.

Utilizing the rank based strategy, the two thumbs case $(1,6)$ appears to be at a significant disadvantage in terms of system matching performance ranking below most of the other cases examined. This disadvantage is marked (i.e., FAP10 two finger $(1,6)$ FNIR $=18.566 \%$ ) and is not substantially mitigated by using various gallery strategies or FAP levels.

Finally, the emerging smaller platen sensors (1/4" square) may present a significant advance in the area of authentication and verification, but their application in one-to-many $(1: \mathrm{N})$ searching for which they are not designed is ill-advised. While the $1 / 4$ " square images perform reasonably well in terms of FPIR rates on the system tested, FNIR performance takes a significant hit where in the worst case scenario the system may fail to match the target identity at a rate of $97.380 \%$ when using two thumbs $(1,6)$. In the best case scenario where all ten fingers can be captured, the experimental 1/4" FAP yields an FNIR performance of $47.432 \%$, with an FPIR of $0.0 \%$. 


\section{Conclusions}

For the typical field case of two finger captured images being matched against a gallery of identities comprised of full FD-249 sets, FAP10 is at a disadvantage compared to other FAPs. In terms of FNIR, FAP10 submissions fail to identify their target almost nearly twice as often as FAP20 (1.164\% vs. $0.506 \%)$ at the system low confidence threshold. For the high confidence threshold, FNIR for FAP10 is again almost twice that of FAP20 $(3.270 \%$ vs. $1.844 \%$ ). FAP30 on the other hand appears to be best/optimal and its performance is on a par with (if not slightly better than) the uncropped control case ( $d=0.0162$ for FAP $30, d=0.0164$ for control).

Comparing various probe finger combinations and Mobile ID FAPs to a gallery of full FD-249 shows stratification of ten and four finger probe sets at the top of the ranking scale (section 4.3.1) and the two finger probe sets occupying most of the lower half of the list. While the FAP10 two finger $(2,7)$ case is not the worst performing among those tested (ranked 14 out of 20), significant matching performance benefits can be realized by either increasing the count of fingers captured in FAP10, or utilizing FAP30 for two finger capture cases.

In examining the impact of gallery configuration on system performance (section 4.4.1), a complete FD-249 gallery emerges as the optimal gallery configuration, with FAP30 ranking as best case tested among the various FAPs on this gallery. Rankings show that performance degradation observed by rolled-only or flat-only gallery configurations can be mitigated with larger FAPs or more fingers. The case of FAP10 ranks last among the Mobile ID FAPs when used on a rolled-only or flat-only gallery.

Throughout all FAPs, finger combinations and database combinations studied, system FPIR rates remained flat at $0 \%$ at the high confidence threshold. For the low confidence threshold, FPIR rates fluctuated slightly across the various test cases examined but generally remained low ranging from $0.028 \%$ to $0.714 \%$.

Comparing the various Mobile ID FAP scenarios (section 4.5.1) for throughput performance shows that system match times appear closely linked to image spatial geometry where median search times for all image search FAPs consistently increases from FAP10 to FAP30 (with certain isolated exceptions). The two finger case of $(2,7)$ appears to provide the best throughput performance out of all cases examined, while the two finger case of $(1,6)$ incurs an unusually large throughput penalty.

Given the data in this study, the typical field case of FAP10 two finger $(2,7)$ suffers from a significant performance penalty in terms of FNIR, showing a miss-rate almost twice as high as FAP20 and FAP30. It is possible to reduce FNIR significantly by adopting a larger capture profile such as FAP20 or FAP30. In cases where investment in legacy equipment must be preserved, a possibility mitigation strategy for this performance gap is to utilize four finger capture (i.e., 2,3,7,8) with the smaller FAP10. It should be noted however that simply capturing more fingers using the smaller single-finger mobile capture devices may introduce risk in terms of sequencing errors during the capture process so this mitigation strategy may introduce risk in terms of potential sequence errors. 


\section{References}

AN2k11 NIST Special Publication 500-290 Update 2013: “American National Standard for Information Systems - Data Format for the Interchange of Fingerprint, Facial \& Other Biometric Information (ANSI/NIST ITL 1-2011)”.

Approved December 2013. http://biometrics.nist.gov/cs_links/standard/ansi_2012/UpdateFinal_Approved_Version.pdf. Retrieved 01/31/2014.

MOBID Orandi, S., McCabe, R. M., “Mobile ID Device Best Practice Recommendation Version 1.0”, NIST SP 500-280, National Institute of Standards and Technology, Gaithersburg, MD. http://www.nist.gov/customcf/get pdf.cfm?pub id=903169. Retrieved 03/11/2013. 
APPENDIX A. AGGREGATE TEST DATA 
Table 16 - Summary Matching Performance Data by Probe and Gallery Type
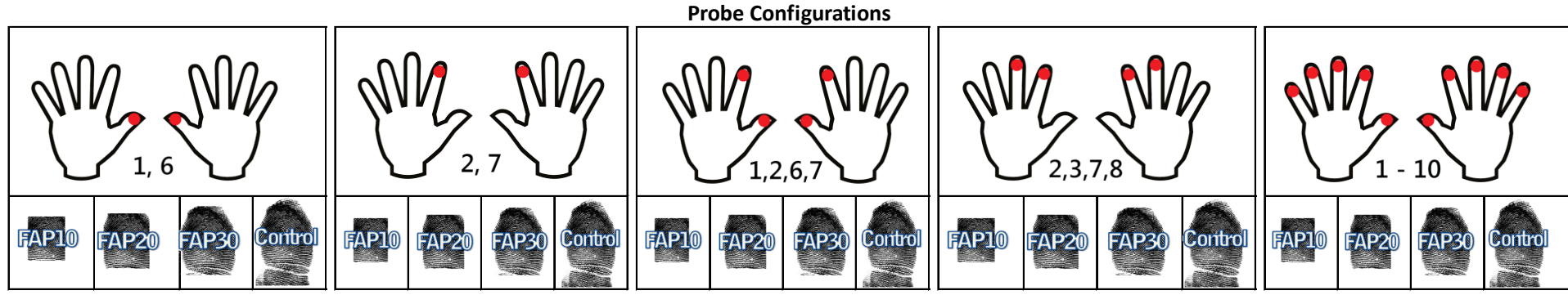

RISC High Confidence System Hit (Threshold=5000)

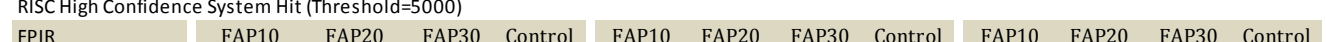

M

1

Q

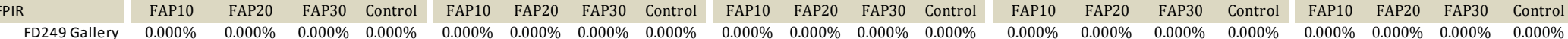

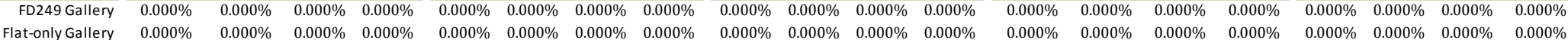
\begin{tabular}{lllllllllllllllllllllll} 
Rolled-onfylery & $0.000 \%$ & $0.000 \%$ & $0.000 \%$ & $0.000 \%$ & $0.000 \%$ & $0.000 \%$ & $0.000 \%$ & $0.000 \%$ & $0.000 \%$ & $0.000 \%$ & $0.000 \%$ & $0.000 \%$ & $0.000 \%$ & $0.000 \%$ & $0.000 \%$ & $0.000 \%$ & $0.000 \%$ & $0.000 \%$ & $0.000 \%$ & $0.000 \%$ \\
\hline
\end{tabular} \begin{tabular}{|l|lllllllllllllllllllll}
\hline FNIR & $0.000 \%$ & $0.000 \%$ & $0.000 \%$ & $0.000 \%$ & $0.000 \%$ & $0.000 \%$ & $0.000 \%$ & $0.000 \%$ & $0.000 \%$ & $0.000 \%$ & $0.000 \%$ & $0.000 \%$ & $0.000 \%$ & $0.000 \%$ & $0.000 \%$ & $0.000 \%$ & $0.000 \%$ & $0.000 \%$ & $0.000 \%$ & $0.000 \%$ \\
\hline
\end{tabular}

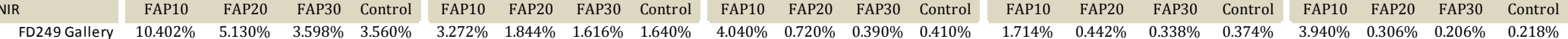

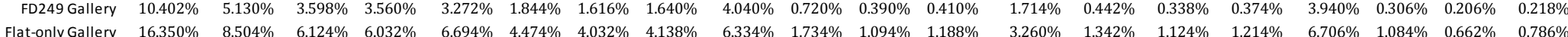

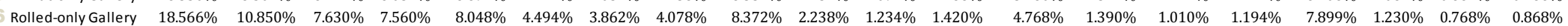

RISC Low Confidence System Hit (Threshold=2000)

$\begin{array}{llllllllllllllllllllll}\text { FPIR } & \text { FAP10 } & \text { FAP20 } & \text { FAP30 } & \text { Control } & \text { FAP10 } & \text { FAP20 } & \text { FAP30 } & \text { Control } & \text { FAP10 } & \text { FAP20 } & \text { FAP30 } & \text { Control } & \text { FAP10 } & \text { FAP20 } & \text { FAP30 } & \text { Control } & \text { FAP10 } & \text { FAP20 } & \text { FAP30 } & \text { Control }\end{array}$ \begin{tabular}{lllllllllllllllllllll} 
FD249 Gallery & $0.208 \%$ & $0.106 \%$ & $0.090 \%$ & $0.046 \%$ & $0.340 \%$ & $0.422 \%$ & $0.356 \%$ & $0.368 \%$ & $0.146 \%$ & $0.128 \%$ & $0.122 \%$ & $0.158 \%$ & $0.622 \%$ & $0.650 \%$ & $0.548 \%$ & $0.546 \%$ & $0.034 \%$ & $0.060 \%$ & $0.054 \%$ & $0.052 \%$ \\
\hline
\end{tabular} $\begin{array}{lllllllllllllllllllll}\text { Flat-only Gallery } & 0.218 \% & 0.132 \% & 0.082 \% & 0.064 \% & 0.374 \% & 0.462 \% & 0.396 \% & 0.406 \% & 0.182 \% & 0.166 \% & 0.138 \% & 0.158 \% & 0.704 \% & 0.664 \% & 0.714 \% & 0.644 \% & 0.036 \% & 0.052 \% & 0.060 \% & 0.066 \%\end{array}$ \begin{tabular}{lllllllllllllllllllll} 
Rolled-only Gallery & $0.176 \%$ & $0.080 \%$ & $0.076 \%$ & $0.054 \%$ & $0.294 \%$ & $0.236 \%$ & $0.222 \%$ & $0.284 \%$ & $0.164 \%$ & $0.104 \%$ & $0.098 \%$ & $0.108 \%$ & $0.462 \%$ & $0.356 \%$ & $0.258 \%$ & $0.344 \%$ & $0.064 \%$ & $0.028 \%$ & $0.062 \%$ & $0.036 \%$ \\
\hline
\end{tabular}

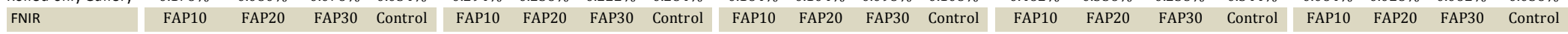
\begin{tabular}{lllllllllllllllllllll}
\hline FD249 Gallery & $5.938 \%$ & $2.620 \%$ & $1.964 \%$ & $1.890 \%$ & $1.164 \%$ & $0.506 \%$ & $0.400 \%$ & $0.472 \%$ & $3.658 \%$ & $0.464 \%$ & $0.216 \%$ & $0.226 \%$ & $1.372 \%$ & $0.236 \%$ & $0.160 \%$ & $0.192 \%$ & $3.740 \%$ & $0.190 \%$ & $0.098 \%$ & $0.134 \%$ \\
\hline
\end{tabular} \begin{tabular}{lllllllllllllllllllll} 
Flat-only Gallery & $11.122 \%$ & $5.268 \%$ & $3.930 \%$ & $3.846 \%$ & $2.806 \%$ & $1.766 \%$ & $1.570 \%$ & $1.726 \%$ & $5.786 \%$ & $1.364 \%$ & $0.796 \%$ & $0.888 \%$ & $2.660 \%$ & $0.936 \%$ & $0.714 \%$ & $0.848 \%$ & $6.438 \%$ & $0.946 \%$ & $0.538 \%$ & $0.686 \%$ \\
\hline
\end{tabular}

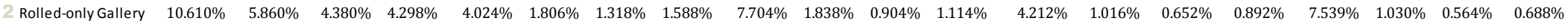

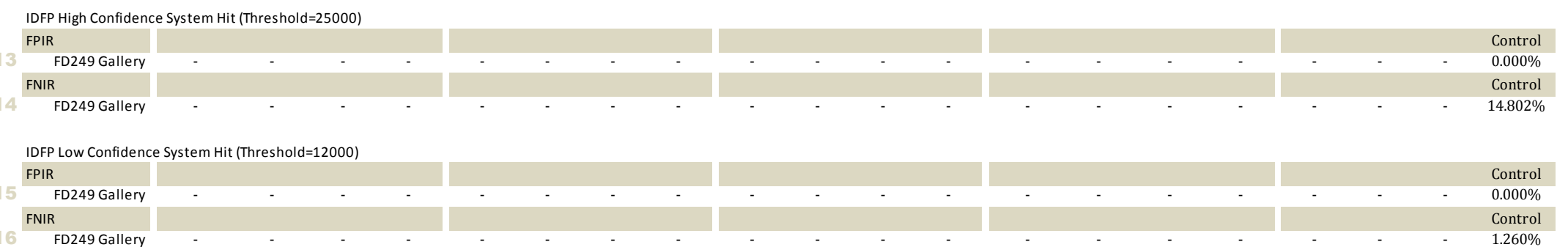


Table 17 - Summary Matching Throughput by Probe and Gallery Type (median match time, milliseconds)

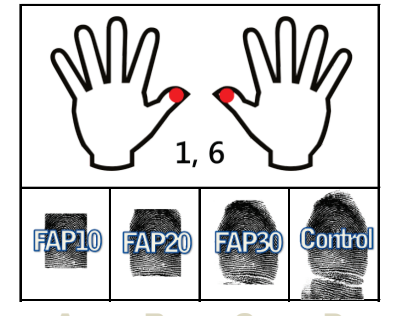

RISC Matcher Configuration

Probe Contents Probe Pairing Gallery FAP10 FAP20 FAP30 Control

1 Image $\quad$ Non-mated $\quad$ FD249 $3068 \quad 3973 \quad 5323 \quad 5445$

2 Image

3 Image

4 Image

5 Image

6 Image

7 Template

8 Template

9 Template

10 Template

1 Template

12 Template
Mated

Non-mated

Mated

Non-mated

Mated

Non-mated

Mated

Non-mated

Mated

Non-mated

Mated $\begin{array}{llllll}\text { FD249 } & 2942 & 3928 & 5345 & 5467\end{array}$

Flat $\quad \begin{array}{lllll}2110 & 2765 & 3716 & 3845\end{array}$

Flat $\quad \begin{array}{lllll}2141 & 2823 & 3813 & 3901\end{array}$

Rolled

$\begin{array}{llll}2327 & 3117 & 4181 & 4291\end{array}$

$\begin{array}{llll}2565 & 3307 & 4424 & 4545\end{array}$

\section{FD249}

FD249

$\begin{array}{llll}1652 & 2074 & 2534 & 2595\end{array}$

$\begin{array}{llll}1566 & 2035 & 2587 & 2625\end{array}$

$\begin{array}{llll}827 & 994 & 1192 & 1211\end{array}$

Flat

$\begin{array}{llll}854 & 1035 & 1246 & 1281\end{array}$

Rolled

Rolled

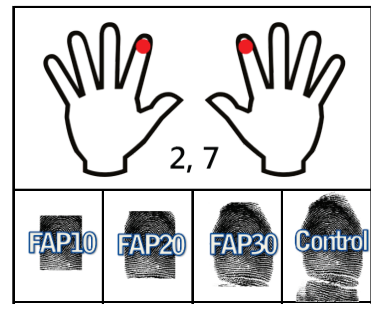
$\begin{array}{llll}1966 & 2370 & 3059 & 2690\end{array}$ $\begin{array}{lllll}1841 & 2251 & 2964 & 2568\end{array}$ $\begin{array}{llll}1696 & 2068 & 2604 & 2338\end{array}$ $\begin{array}{lllll}1697 & 2074 & 2610 & 2344\end{array}$ $\begin{array}{llll}1681 & 2061 & 2665 & 2350\end{array}$ $\begin{array}{llll}1772 & 2159 & 2764 & 2439\end{array}$

\section{$\begin{array}{llll}763 & 807 & 936 & 851\end{array}$}

$\begin{array}{llll}663 & 707 & 870 & 756\end{array}$

$\begin{array}{llll}551 & 564 & 616 & 605\end{array}$

$\begin{array}{llll}547 & 564 & 612 & 599\end{array}$

$\begin{array}{llll}639 & 645 & 745 & 701\end{array}$

$\begin{array}{llll}625 & 638 & 739 & 682\end{array}$

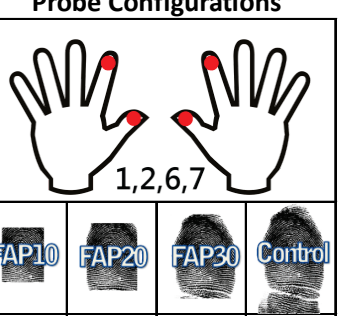

FAP10 FAP20 FAP30 Control $\begin{array}{llll}2155 & 2675 & 3713 & 3675\end{array}$ $\begin{array}{lllll}1945 & 2505 & 3530 & 3481\end{array}$

$\begin{array}{llll}1883 & 2364 & 3213 & 3252\end{array}$

$\begin{array}{llll}1908 & 2398 & 3276 & 3299\end{array}$

$\begin{array}{llll}1782 & 2269 & 3140 & 3168\end{array}$

$\begin{array}{llll}2044 & 2504 & 3450 & 3379\end{array}$

$\begin{array}{llll}827 & 879 & 1037 & 979\end{array}$

$\begin{array}{llll}687 & 770 & 969 & 881\end{array}$

$\begin{array}{llll}580 & 623 & 706 & 700\end{array}$

$\begin{array}{llll}592 & 640 & 740 & 722\end{array}$

$\begin{array}{llll}725 & 747 & 838 & 817\end{array}$

$\begin{array}{llll}724 & 752 & 855 & 828\end{array}$

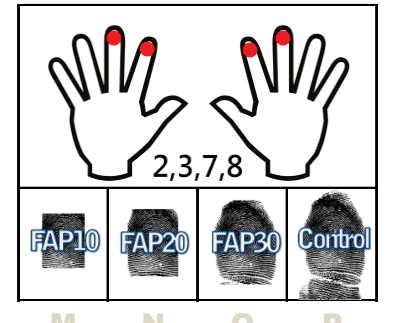

FAP10 FAP20 FAP30 Control $\begin{array}{llll}2205 & 2664 & 3518 & 3119\end{array}$

$\begin{array}{llll}1996 & 2483 & 3327 & 2935\end{array}$

$\begin{array}{llll}1892 & 2296 & 2950 & 2731\end{array}$

$\begin{array}{llll}1849 & 2290 & 2993 & 2711\end{array}$

$\begin{array}{llll}1807 & 2239 & 2931 & 2640\end{array}$

$\begin{array}{llll}1945 & 2357 & 3082 & 2841\end{array}$

$\begin{array}{llll}853 & 898 & 1090 & 977\end{array}$

$\begin{array}{llll}745 & 914 & 1125 & 842\end{array}$

$\begin{array}{llll}614 & 644 & 732 & 698\end{array}$

$\begin{array}{llll}614 & 650 & 741 & 704\end{array}$

$\begin{array}{llll}740 & 747 & 851 & 799\end{array}$

$\begin{array}{llll}722 & 728 & 849 & 804\end{array}$

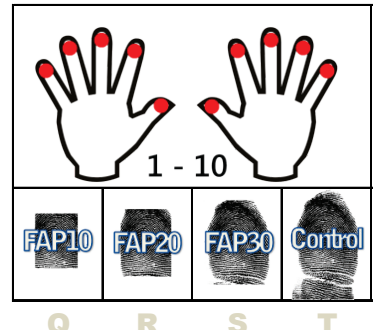

FAP10 FAP20 FAP30 Control $\begin{array}{llll}3377 & 3671 & 4400 & 4336\end{array}$ $\begin{array}{lllll}2050 & 2537 & 3426 & 3362\end{array}$ $\begin{array}{llll}2093 & 2713 & 3573 & 3197\end{array}$ $\begin{array}{lllll}2177 & 2806 & 3569 & 3389\end{array}$ $\begin{array}{llll}2085 & 2543 & 3331 & 3340\end{array}$ $\begin{array}{llll}3073 & 3334 & 4107 & 4043\end{array}$

$\begin{array}{llll}1816 & 1506 & 1536 & 2099\end{array}$ $\begin{array}{llll}1258 & 1243 \quad 993 & 1293\end{array}$ $\begin{array}{llll}1088 & 900 \quad 842 & 1888\end{array}$ $\begin{array}{llll}904 & 791 & 834 & 1155\end{array}$ $\begin{array}{llll}1295 & 1088 & 1181 & 1338\end{array}$ $\begin{array}{llll}1158 & 1074 & 1193 & 1594\end{array}$

IDFP Matcher Configuration

$\begin{array}{lll}13 \text { Image } & \text { Non-mated } & \text { FD249 } \\ 14 \text { Image } & \text { Mated } & \text { FD249 } \\ & & \\ 15 \text { Template } & \text { Non-mated } & \text { FD249 } \\ 16 \text { Template } & \text { Mated } & \text { FD249 }\end{array}$

